Supporting Information

\title{
DFT Studies of Alkene Insertions into Cu-B Bonds in Copper(I) Boryl Complexes
}

\author{
Li Dang, Haitao Zhao and Zhenyang Lin* \\ Department of Chemistry, The Hong Kong University of Science and Technology, Clear \\ Water Bay, Kowloon, Hong Kong \\ Todd B. Marder* \\ Department of Chemistry, Durham University, South Road, Durham DH1 3LE, UK
}

Author Email Addresses: chzlin@ust.hk and todd.marder@durham.ac.uk

\section{Complete Reference for Ref (17):}

Frisch, M. J.; Trucks, G. W.; Schlegel, H. B.; Scuseria, G. E.; Robb, M. A.; Cheeseman, J. R.; Montgomery, J. A.; Vreven, T., Jr.; Kudin, K. N.; Burant, J. C.; Millam, J. M.; Iyengar, S. S.; Tomasi, J.; Barone, V.; Mennucci, B.; Cossi, M.; Scalmani, G.; Rega, N.; Petersson, G. A.; Nakatsuji, H.; Hada, M.; Ehara, M.; Toyota, K.; Fukuda, R.; Hasegawa, J.; Ishida, M.; Nakajima, T.; Honda, Y.; Kitao, O.; Nakai, H.; Klene, M.; Li, X.; Knox, J. E.; Hratchian, H. P.; Cross, J. B.; Adamo, C.; Jaramillo, J.; Gomperts, R.; Stratmann, R. E.; Yazyev, O.; Austin, A. J.; Cammi, R.; Pomelli, C.; Ochterski, J. W.; Ayala, P. Y.; Morokuma, K.; Voth, G. A.; Salvador, P.; Dannenberg, J. J.; Zakrzewski, V. G.; Dapprich, S.; Daniels, A. D.; Strain, M. C.; Farkas, O.; Malick, D. K.; Rabuck, A. D.; Raghavachari, K.; Foresman, J. B.; Ortiz, J. V.; Cui, Q.; Baboul, A. G.; Clifford, S.; Cioslowski, J.; Stefanov, B. B.; Liu, G.; Liashenko, A.; Piskorz, P.; Komaromi, I.; Martin, R. L.; Fox, D. J.; Keith, T.; Al-Laham, M. A.; Peng, C. Y.; Nanayakkara, A.; Challacombe, M.; Gill, P. M. W.; Johnson, B.; Chen, W.; Wong, M. W.; Gonzalez, C.; Pople, J. A. Gaussian 03, revision B05; Gaussian, Inc.: Pittsburgh, PA, 2003. 


\section{Cartesian coordinates for all the complexes calculated in this study:}

\section{$\mathrm{CH}_{2} \mathrm{CHPh}$}

$\begin{array}{crrr}\text { E }=-309.615334591 \text { au } & \text { ZPE }=84.4 \mathrm{kcal} / \mathrm{mol} \\ \mathrm{C} & -1.95933600 & -0.52770100 & -0.00038900 \\ \mathrm{C} & -2.97972200 & 0.33411000 & 0.00054500 \\ \mathrm{C} & -0.51612100 & -0.21968200 & -0.00024700 \\ \mathrm{C} & 0.40548700 & -1.28589600 & 0.00001000 \\ \mathrm{C} & 1.78331200 & -1.05046400 & 0.00024800 \\ \mathrm{C} & 2.26922500 & 0.26117400 & 0.00020300 \\ \mathrm{C} & 1.36530300 & 1.33327500 & -0.00010700 \\ \mathrm{C} & -0.00961700 & 1.09694400 & -0.00034400 \\ \mathrm{H} & -2.19267800 & -1.59375100 & -0.00134000 \\ \mathrm{H} & -4.00447800 & -0.01686400 & 0.00025400 \\ \mathrm{H} & -2.83981500 & 1.41270300 & 0.00159500 \\ \mathrm{H} & 0.03200800 & -2.30609800 & 0.00005200 \\ \mathrm{H} & 2.47406600 & -1.88783900 & 0.00044400 \\ \mathrm{H} & 3.33824100 & 0.44863700 & 0.00036000 \\ \mathrm{H} & 1.73528500 & 2.35390400 & -0.00019400 \\ \mathrm{H} & -0.69381500 & 1.93874600 & -0.00068800\end{array}$

$1 \mathrm{~A}$

$\begin{array}{crrr}\text { E }=-2199.16446218 a u & \text { ZPE }=125.5 \mathrm{kcal} / \mathrm{mol} \\ \mathrm{Cu} & 0.00000000 & 0.00000000 & 0.00000000 \\ \mathrm{~B} & 1.97934800 & 0.00000000 & 0.00000000 \\ \mathrm{O} & 2.77999235 & 1.14410205 & 0.00000000 \\ \mathrm{O} & 2.77999365 & -1.14410105 & 0.00000000 \\ \mathrm{C} & -1.90816800 & 0.00000085 & 0.00000000 \\ \mathrm{~N} & -2.76084140 & -1.07678080 & -0.08406387 \\ \mathrm{~N} & -2.76083559 & 1.07678191 & 0.08412036 \\ \mathrm{C} & -4.10167162 & -0.67922431 & -0.05300358 \\ \mathrm{C} & -4.10166737 & 0.67922503 & 0.05315189 \\ \mathrm{C} & 4.21363258 & 0.76986390 & 0.00098490 \\ \mathrm{C} & 4.21363442 & -0.76986053 & -0.00094446 \\ \mathrm{C} & -2.29150536 & 2.45903013 & 0.19235359 \\ \mathrm{C} & -2.29151864 & -2.45902868 & -0.19232536 \\ \mathrm{H} & -2.63758157 & -2.91250504 & -1.12580757 \\ \mathrm{H} & -4.91730724 & -1.37984345 & -0.10778799 \\ \mathrm{H} & -4.91729976 & 1.37984569 & 0.10799191 \\ \mathrm{H} & 4.68321149 & -1.19998686 & 0.88827311 \\ \mathrm{H} & 4.68151680 & -1.19764025 & -0.89223277 \\ \mathrm{H} & 4.68149319 & 1.19765980 & 0.89227686 \\ \mathrm{H} & 4.68323051 & 1.19997677 & -0.88822917 \\ \mathrm{H} & -2.63750442 & 2.91250249 & 1.12586076 \\ \mathrm{H} & -1.20074735 & 2.43286826 & 0.18607969 \\ \mathrm{H} & -2.64419287 & 3.05409250 & -0.65512407 \\ \mathrm{H} & -1.20076065 & -2.43286680 & -0.18612614 \\ \mathrm{H} & -2.64414712 & -3.05408808 & 0.65517773\end{array}$

\section{IN1}

$\begin{array}{crrr}\text { E }=-2508.80265542 & \text { au ZPE }=211.3 \mathrm{kcal} / \mathrm{mol} \\ \mathrm{C} & 0.00000000 & 0.00000000 & 0.00000000 \\ \mathrm{C} & 2.51507822 & 0.00000000 & 0.00000000 \\ \mathrm{C} & -0.30771292 & 2.27508498 & 0.00000000 \\ \mathrm{C} & 0.02385386 & -2.89367703 & 1.60839299 \\ \mathrm{C} & -0.59143283 & -3.69095692 & 0.62219880 \\ \mathrm{C} & 1.03420490 & 2.04934812 & -0.00708693 \\ \mathrm{C} & 1.46516954 & -2.88563955 & 1.91494573 \\ \mathrm{C} & 1.93324731 & -2.18049054 & 3.04809948 \\ \mathrm{C} & 3.29222363 & -2.12650061 & 3.36305989 \\ \mathrm{C} & 4.23392739 & -2.77441126 & 2.55091951 \\ \mathrm{C} & 3.78918714 & -3.48506273 & 1.42837857 \\ \mathrm{C} & 2.42865657 & -3.54317805 & 1.11415804 \\ \mathrm{C} & -2.37499369 & 0.79922665 & -0.03772859 \\ \mathrm{Cu} & -0.41870953 & -1.88423109 & -0.14559968 \\ \mathrm{~N} & -0.92552769 & 1.01994416 & 0.00024060 \\ \mathrm{~N} & 1.20722815 & 0.65969440 & -0.00746815 \\ \mathrm{H} & 2.92863887 & -0.04143665 & 1.01100610 \\ \mathrm{H} & 2.40140739 & -1.01909977 & -0.36663348 \\ \mathrm{H} & 3.20021321 & 0.54208258 & -0.65706901\end{array}$

$\begin{array}{rrr}-0.86816282 & 3.19399682 & -0.00382170 \\ -0.60881769 & -2.49336294 & 2.40064035 \\ -1.64256268 & -3.94696080 & 0.73802482 \\ -0.02205666 & -4.41802670 & 0.05553840 \\ 1.86462505 & 2.73408301 & -0.01756756 \\ 1.21145789 & -1.67421817 & 3.68345237 \\ 3.61827847 & -1.58303146 & 4.24551121 \\ 5.29084925 & -2.73574832 & 2.79492111 \\ 4.50539142 & -4.00215023 & 0.79603003 \\ 2.11146368 & -4.10441706 & 0.24137772 \\ -2.86887376 & 1.73927029 & -0.29304503 \\ -2.73912326 & 0.46168356 & 0.93739511 \\ -2.60999206 & 0.04770494 & -0.79669362 \\ -1.18218439 & -2.18991752 & -1.97724122 \\ -2.44086953 & -1.70912208 & -2.35276991 \\ -0.57030020 & -2.83514005 & -3.04165398 \\ -2.73031636 & -2.09062606 & -3.76107108 \\ -1.44543879 & -2.79019697 & -4.24069009 \\ -3.60330849 & -2.74876453 & -3.76399377 \\ -2.96295924 & -1.18246077 & -4.32277397 \\ -1.61459002 & -3.81516367 & -4.57924451 \\ -0.92342618 & -2.23506127 & -5.02481711\end{array}$

TS1

$\mathrm{E}=-2508.78046714 \mathrm{au} \quad \mathrm{ZPE}=210.7 \mathrm{kcal} / \mathrm{mol}$

$\mathrm{Cu}=0.00000000 \quad 0.00000000 \quad 0.00000000$

1.97970682

$\begin{array}{lll}1.10358180 & 1.69244208 & 0.00000000\end{array}$

$\begin{array}{lll}1.44385115 & -1.37072572 & 0.29508738\end{array}$

$\begin{array}{lll}-2.14300165 & 1.40272207 & -2.22146481\end{array}$

$\begin{array}{lll}1.23723917 & 2.45436062 & 1.15407994\end{array}$

$1.23696923 \quad 2.47352970 \quad-1.14631959$

$\begin{array}{lll}-1.85289167 & 0.19999494 & -0.04199169\end{array}$

$\begin{array}{lll}-2.65335728 & 0.79955338 & -0.99021414\end{array}$

$\begin{array}{lll}-2.74429466 & -0.23195842 & 0.91559528\end{array}$

$\begin{array}{lll}-4.00405218 & 0.73108093 & -0.63148689\end{array}$

$\begin{array}{lll}-4.05944071 & 0.09128236 & 0.56958515\end{array}$

$\begin{array}{lll}1.39366140 & 3.87673977 & 0.78541347\end{array}$

$\begin{array}{lll}1.57147567 & 3.86171799 & -0.74817028\end{array}$

$\begin{array}{lll}-2.33547461 & -0.92983650 & 2.13716074\end{array}$

$\begin{array}{lll}-4.78703956 & 1.13184311 & -1.25184498\end{array}$

$\begin{array}{lll}-4.89868637 & -0.15761190 & 1.19582931\end{array}$

$\begin{array}{lll}2.60039000 & 4.06565593 & -1.05751532\end{array}$

$\begin{array}{lll}0.89681116 & 4.54501791 & -1.26817866\end{array}$

$\begin{array}{lll}2.25998665 & 4.27844983 & 1.31518174\end{array}$

$\begin{array}{lll}0.49584574 & 4.41552203 & 1.10150320\end{array}$

$\begin{array}{lll}-1.43368427 & -1.50716935 & 1.92589958\end{array}$

$\begin{array}{lll}-2.11678533 & -0.22022431 & 2.94077902\end{array}$

$\begin{array}{lll}-3.13254103 & -1.60559934 & 2.45470388\end{array}$

$\begin{array}{lll}-1.12335395 & 1.75348357 & -2.04648520\end{array}$

$\begin{array}{lll}-2.13730567 & 0.67688546 & -3.04142519\end{array}$

$\begin{array}{lll}-2.77302054 & 2.25113020 & -2.50030694\end{array}$

$\begin{array}{lll}2.42737736 & 0.06894307 & -0.98953045\end{array}$

$\begin{array}{lll}2.66366096 & 0.38448887 & 0.76348650\end{array}$

$\begin{array}{lll}1.45690494 & -1.89365610 & 1.66011220\end{array}$

$\begin{array}{lll}1.54694794 & -1.05599110 & 2.80685056\end{array}$

$\begin{array}{lll}1.51962017 & -1.58873089 & 4.09908333\end{array}$

$\begin{array}{lll}1.39974518 & -2.96810237 & 4.30862690\end{array}$

$\begin{array}{lll}1.34000665 & -3.29039352 & 1.89899332\end{array}$

$\begin{array}{lll}1.31143083 & -3.81281347 & 3.19031407\end{array}$

$\begin{array}{lll}1.63650565 & 0.01989187 & 2.68298837\end{array}$

$\begin{array}{lll}1.22772351 & -4.88786195 & 3.32959308\end{array}$

$\begin{array}{lll}1.59679575 & -2.10543988 & -0.49256487\end{array}$

$\begin{array}{lll}1.59579778 & -0.91694348 & 4.95078754\end{array}$

$\begin{array}{lll}1.38223839 & -3.37707003 & 5.31402983\end{array}$

$\begin{array}{lll}1.27531621 & -3.95936922 & 1.04455699\end{array}$ 
2A

$\mathrm{E}=-2508.82558602 \mathrm{au} \quad \mathrm{ZPE}=212.1 \mathrm{kcal} / \mathrm{mol}$

$\begin{array}{lrrr}\mathrm{B} & 0.00000000 & 0.00000000 & 0.00000000 \\ \mathrm{C} & 4.77222462 & 0.00000000 & 0.00000000 \\ \mathrm{C} & 6.30759141 & 1.97223845 & 0.00000000 \\ \mathrm{C} & 6.35271791 & -1.65709737 & -0.11689101 \\ \mathrm{C} & 1.53496463 & 2.00444702 & 0.39026643 \\ \mathrm{C} & 0.43130700 & 1.10314482 & 1.00941091 \\ \mathrm{C} & 7.01575395 & -0.46666594 & -0.11465055 \\ \mathrm{C} & 1.88937235 & 3.21765671 & 1.19806830 \\ \mathrm{C} & 2.45743605 & 4.35072158 & 0.56170192 \\ \mathrm{C} & 2.84212341 & 5.48328936 & 1.27981711 \\ \mathrm{C} & 2.67266062 & 5.53435386 & 2.67168229 \\ \mathrm{C} & 2.10272760 & 4.43355339 & 3.32004130 \\ \mathrm{C} & 1.71333793 & 3.29899887 & 2.59819460 \\ \mathrm{C} & -1.14026748 & -1.05899720 & -1.73703065 \\ \mathrm{C} & -0.04984183 & -2.01226428 & -1.18971849 \\ \mathrm{C} & 3.91284382 & -2.35096825 & -0.00604160 \\ \mathrm{Cu} & 3.15772137 & 0.95675998 & 0.14282890 \\ \mathrm{~N} & 4.98821624 & -1.35677687 & -0.04610246 \\ \mathrm{~N} & 6.04075273 & 0.53267025 & -0.04216659 \\ \mathrm{O} & -1.03100880 & 0.14654562 & -0.90262625 \\ \mathrm{O} & 0.61341465 & -1.24344429 & -0.11971050 \\ \mathrm{H} & 6.78759953 & 2.30428080 & -0.92533741 \\ \mathrm{H} & 5.34878445 & 2.48039637 & 0.11382277 \\ \mathrm{H} & 6.94691271 & 2.21876649 & 0.85231238 \\ \mathrm{H} & 6.72229847 & -2.66737408 & -0.15869096 \\ \mathrm{H} & 1.18562057 & 2.35207530 & -0.59461885 \\ \mathrm{H} & -0.45163046 & 1.69572449 & 1.30576907 \\ \mathrm{H} & 0.80046016 & 0.61372460 & 1.91878566 \\ \mathrm{H} & 8.06920786 & -0.24841461 & -0.15478761 \\ \mathrm{H} & 2.58580437 & 4.32826521 & -0.51855482 \\ \mathrm{H} & 3.26682795 & 6.33484007 & 0.75356176 \\ \mathrm{H} & 2.96913442 & 6.41536786 & 3.23288708 \\ \mathrm{H} & 1.95169914 & 4.45815520 & 4.39654954 \\ \mathrm{H} & 1.26739907 & 2.46355817 & 3.12800264 \\ \mathrm{H} & -0.97272046 & -0.77550566 & -2.77912397 \\ \mathrm{H} & -2.15023096 & -1.46260426 & -1.63345528 \\ \mathrm{H} & -0.46163986 & -2.92569920 & -0.75290338 \\ \mathrm{H} & 0.69776878 & -2.27875119 & -1.94154155 \\ \mathrm{H} & 4.03089197 & -3.00282197 & 0.86478821 \\ & 3.92359226 & -2.95931591 & -0.91592150 \\ \mathrm{H} & 2.95850646 & -1.82582696 & 0.06698420\end{array}$

\section{TS2}

$\mathrm{E}=-2508.78007332 \mathrm{au} \quad \mathrm{ZPE}=208.9 \mathrm{kcal} / \mathrm{mol}$

$\begin{array}{lrrr}\mathrm{B} & 0.00000000 & 0.00000000 & 0.00000000 \\ \mathrm{C} & 4.78236873 & 0.00000000 & 0.00000000 \\ \mathrm{C} & 5.73736302 & 2.31679843 & 0.00000000 \\ \mathrm{C} & 6.72508902 & -1.15714812 & -0.38911207 \\ \mathrm{C} & 1.68322539 & 1.93427941 & -0.10315406 \\ \mathrm{C} & 1.06520218 & 0.86754845 & 0.71213274 \\ \mathrm{C} & 7.04262272 & 0.16714899 & -0.34486602 \\ \mathrm{C} & 2.05267864 & 3.23132289 & 0.45769986 \\ \mathrm{C} & 2.25151704 & 4.34695694 & -0.40196932 \\ \mathrm{C} & 2.62773853 & 5.59594698 & 0.08985907 \\ \mathrm{C} & 2.82526439 & 5.79615735 & 1.46475245 \\ \mathrm{C} & 2.63596532 & 4.71278454 & 2.33246430 \\ \mathrm{C} & 2.26255105 & 3.45777411 & 1.84552255 \\ \mathrm{C} & -1.97945906 & -1.13810194 & -0.41419628 \\ \mathrm{C} & -1.06098383 & -1.23089821 & -1.65919668 \\ \mathrm{C} & 4.58025058 & -2.48898969 & -0.13315237 \\ \mathrm{Cu} & 2.99424469 & 0.44000492 & 0.32006862 \\ \mathrm{~N} & 5.34709843 & -1.24523246 & -0.17335946 \\ \mathrm{~N} & 5.85154884 & 0.85986950 & -0.11368123 \\ \mathrm{O} & -1.22023912 & -0.32002609 & 0.54856450 \\ \mathrm{O} & 0.16896033 & -0.51995480 & -1.26734122 \\ \mathrm{H} & 6.36037194 & 2.79576445 & -0.75943775 \\ \mathrm{H} & 4.69387875 & 2.59156796 & -0.15829911 \\ \mathrm{H} & 6.04645187 & 2.65748298 & 0.99262165 \\ \mathrm{H} & 1.43404211 & 1.93160973 & -1.16269739 \\ \mathrm{H} & 0.76362812 & 1.16341174 & 1.71735774 \\ \mathrm{H} & 1.87865819 & -0.28487533 & 1.21068362 \\ & & & \\ & & & \end{array}$

$\begin{array}{rrr}2.09214495 & 4.21400318 & -1.46921013 \\ 2.76072351 & 6.42380576 & -0.60239565 \\ 3.11293342 & 6.77021458 & 1.84765269 \\ 2.78086906 & 4.84497090 & 3.40196191 \\ 2.13332068 & 2.63940554 & 2.54820582 \\ -2.92789772 & -0.63671912 & -0.61924452 \\ -2.17813655 & -2.11079161 & 0.04264479 \\ -0.79878443 & -2.25870744 & -1.92118837 \\ -1.48197405 & -0.73275986 & -2.53599973 \\ 5.08130912 & -3.22126909 & 0.50587572 \\ 4.45476084 & -2.90604775 & -1.13711929 \\ 3.59891584 & -2.24943971 & 0.28303220 \\ 7.98937179 & 0.66706684 & -0.45620002 \\ 7.34226947 & -2.02318083 & -0.55566711\end{array}$

IN2

$\mathrm{E}=-2508.80974362 \mathrm{au} \quad \mathrm{ZPE}=210.2 \mathrm{kcal} / \mathrm{mol}$

$0.00000000 \quad 0.00000000$

$\begin{array}{llll}\text { C } & 6.27907905 & 0.62290492 & 0.00000000\end{array}$

C $\quad 0.40873582-0.76930764 \quad 3.44639294$

$\begin{array}{llll}\text { C } & 5.24938235 & 2.67196140 & 0.76162983\end{array}$

$\begin{array}{llll}\text { C } & 3.99920531 & 2.04678492 & 0.75781243\end{array}$

C $\quad-0.81430444 \quad-1.62478291 \quad 5.19155766$

$\begin{array}{llll}\text { C } & 3.86418432 & 0.69637706 & 0.37875609\end{array}$

$\begin{array}{llll}\text { C } & -1.22633252 & -0.34111973 & \mathbf{4 . 9 9 9 6 1 1 9 4}\end{array}$

C $\quad 0.89450212 \quad-3.14356586 \quad 4.10709451$

$\begin{array}{llll}\text { C } & -2.26556736 & -0.21026012 & -0.50760227\end{array}$

$\begin{array}{llll}\text { C } & -1.52571375 & -1.51617225 & -0.89039959\end{array}$

$\begin{array}{llll}\text { C } & -0.64244139 & 1.51556668 & 3.38147754\end{array}$

$\begin{array}{llll}\mathrm{Cu} & 1.77654276 & -0.62532080 & 2.06613327\end{array}$

$\begin{array}{lrrr}\mathrm{N} & -0.47784193 & 0.16452461 & 3.93274864\end{array}$

$\begin{array}{llll}\mathrm{N} & 0.17998294 & -1.87101866 & 4.24119651\end{array}$

$\begin{array}{llll}0 & -1.23752246 & 0.62688812 & 0.12968162\end{array}$

$\begin{array}{llll}0 & -0.10893198 & -1.23423607 & -0.62125946\end{array}$

$\begin{array}{llll}\text { C } & 1.29770509 & 0.60131631 & 0.51269475\end{array}$

$\begin{array}{llll}\text { C } & 2.55973034 & -0.01181868 & 0.33884381\end{array}$

$\begin{array}{lllr}\mathrm{C} & 2.55973034 & -0.01181868 & 0.33884381 \\ \mathrm{H} & \mathbf{4 . 9 4 5 5 2 5 2 9} & -1.04429360 & -0.28550623\end{array}$

$\begin{array}{llll}\mathrm{H} & 7.16063617 & 0.06252954 & -0.29689490\end{array}$

$\begin{array}{llll}\mathrm{H} & 7.36671184 & 2.45269938 & 0.38413472\end{array}$

$\begin{array}{llll}\mathrm{H} & 5.32675477 & 3.71463188 & 1.05613387\end{array}$

$\begin{array}{llll}\mathrm{H} & 3.12246441 & 2.61859832 & 1.04301836\end{array}$

$\begin{array}{rrrr}\mathrm{H} & -1.12899458 & -2.36696213 & 5.90472692 \\ \mathrm{H} & -1.96471327 & 0.24666027 & 5.51713530\end{array}$

$\begin{array}{rrr}-1.96471327 & 0.24666027 & 5.51713530 \\ 0.26346626 & -3.89164173 & 3.61698401\end{array}$

$\begin{array}{llll}\mathrm{H} & 0.26346626 & -3.89164173 & 3.61698401 \\ \mathrm{H} & 1.19022738 & -3.50720091 & 5.09471127 \\ \mathrm{H} & 1.78341564 & -2.95425921 & 3.50085735\end{array}$

$\begin{array}{lrrr}\mathrm{H} & -3.07828185 & -0.37347993 & 0.20452409\end{array}$

$\begin{array}{llll}\mathrm{H} & -2.65517597 & 0.32389044 & -1.37877354\end{array}$

$\begin{array}{llll}\mathrm{H} & -1.83277377 & -2.36911300 & -0.27802070\end{array}$

$\begin{array}{llll}\mathrm{H} & -1.63645022 & -1.77577830 & -1.94561158\end{array}$

$\mathrm{H} \quad-1.37480914 \quad 2.05221306 \quad 3.98802823$

$\begin{array}{llll}\mathrm{H} & -0.99078147 & 1.45864540 & 2.34785791\end{array}$

$\begin{array}{lrrr}\mathrm{H} & 0.30694515 & 2.05359504 & 3.41628398\end{array}$

H $\quad 2.95530736 \quad-1.51698441 \quad 2.52898091$

H $\quad \begin{array}{llll}2.58325865 & -0.92172119 & -0.26235596\end{array}$

H $\quad 1.26645212 \quad 1.64351398 \quad 0.83019276$

TS3

$\begin{array}{crrr}\text { E }=-2508.78802001 & \text { au } & \text { ZPE }=209.2 \mathrm{kcal} / \mathrm{mol} \\ \text { B } & 0.00000000 & 0.00000000 & 0.00000000 \\ \text { C } & 4.64316802 & 0.00000000 & 0.00000000 \\ \text { C } & 5.89491403 & 0.62548925 & 0.00000000 \\ \text { C } & 6.24802273 & 1.49366828 & 1.03925066 \\ \text { C } & -0.22081413 & -0.62067882 & 3.89723790 \\ \text { C } & 5.34058677 & 1.72704788 & 2.08041996 \\ \text { C } & 4.09317264 & 1.09638779 & 2.07982466 \\ \text { C } & -1.33101928 & -1.22314866 & 5.81267516 \\ \text { C } & 3.72361565 & 0.22982303 & 1.03730019 \\ \text { C } & -2.02177730 & -0.19255342 & 5.24892514 \\ \text { C } & 0.76965668 & -2.50669745 & 5.22009361\end{array}$ 


\begin{tabular}{|c|c|c|c|}
\hline $\mathrm{C}$ & -2.05683977 & 0.03070609 & -1.10150035 \\
\hline C & -1.53207917 & -1.42124127 & -1.04303902 \\
\hline C & -1.74951925 & 1.25120998 & 3.18967723 \\
\hline $\mathrm{Cu}$ & 1.04368928 & -0.53804412 & 2.50057456 \\
\hline $\mathrm{N}$ & -1.33813914 & 0.16116693 & 4.08356617 \\
\hline $\mathrm{N}$ & -0.23761398 & -1.47143846 & 4.98118740 \\
\hline & -1.14015552 & 0.78633170 & -0.24146861 \\
\hline & -0.17422477 & -1.29614954 & -0.50769715 \\
\hline C & 1.21933101 & 0.46718925 & 0.7301545 \\
\hline C & 2.36574545 & -0.41014181 & 0.98002930 \\
\hline $\mathrm{H}$ & 4.36933611 & -0.66623584 & -0.8128062 \\
\hline $\mathrm{H}$ & 6.59226551 & 0.43470831 & -0.81047682 \\
\hline $\mathrm{H}$ & 7.21914470 & 1.97919190 & 1.04104556 \\
\hline & 5.60850591 & 2.39245850 & 2.89602122 \\
\hline & 3.39965632 & 1.26226551 & 2.89944750 \\
\hline $\mathrm{H}$ & -1.51999804 & -1.79059143 & 6.70760733 \\
\hline $\mathrm{H}$ & -2.91977240 & 0.30904301 & 5.56606124 \\
\hline $\mathrm{H}$ & 0.32839124 & -3.50364701 & 5.12921894 \\
\hline & 1.20485530 & -2.38901107 & 6.21641600 \\
\hline $\mathrm{H}$ & 1.54545749 & -2.38086241 & 4.462054 \\
\hline $\mathrm{H}$ & -3.07555739 & 0.13464711 & -0.7183932 \\
\hline & -2.01445882 & 0.44613347 & -2.1141131 \\
\hline $\mathrm{H}$ & -2.12378520 & -2.04991663 & -0.367662 \\
\hline $\mathrm{H}$ & -1.48592778 & -1.90017722 & -2.0242118 \\
\hline $\mathrm{H}$ & -2.84087430 & 1.29896572 & 3.1619414 \\
\hline & -1.37054781 & 1.06098497 & 2.1850799 \\
\hline 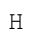 & -1.35697441 & 2.20859916 & 3.5457518 \\
\hline & 2.47548404 & -1.20090467 & 2.3449021 \\
\hline & 2.35649845 & -1.31907084 & 0.3779613 \\
\hline & 1.36070022 & 1.53053365 & 0.9205773 \\
\hline
\end{tabular}

\section{A}

$\mathrm{E}=-2508.83337713 \mathrm{au} \quad \mathrm{ZPE}=212.4 \mathrm{kcal} / \mathrm{mol}$

\begin{tabular}{|c|c|c|c|}
\hline \\
\hline & 0.00000000 & 0.00000000 & 0.00000000 \\
\hline C & 1.51200736 & 0.00000000 & 0.00000000 \\
\hline & 3.37677858 & 2.95479287 & 0.00000000 \\
\hline C & 2.23383588 & 0.91589540 & 1.00842524 \\
\hline C & 3.38412207 & 4.29699215 & -0.39882933 \\
\hline C & 2.25545806 & 5.09676787 & -0.18890329 \\
\hline & 1.66044011 & -3.80803804 & 0.47486384 \\
\hline c & 1.12210200 & 4.54103138 & 0.41985795 \\
\hline & 1.11663444 & 3.19906866 & 0.81299334 \\
\hline & 2.18431572 & -5.96597650 & 1.02317627 \\
\hline c & 2.24671314 & 2.38377449 & 0.60984314 \\
\hline C & 1.12459358 & -6.00951292 & 0.17094409 \\
\hline c & 3.58116246 & -4.10000330 & 2.03833690 \\
\hline & -2.23255791 & -0.29964083 & -0.72048691 \\
\hline & -2.23244424 & 0.11199020 & 0.76911077 \\
\hline & -0.26028108 & -4.27089027 & -1.06150077 \\
\hline & 1.64141968 & -1.92043394 & 0.30960789 \\
\hline $\mathrm{N}$ & 0.81837652 & -4.68287512 & -0.15299967 \\
\hline & 2.49747987 & -4.61267031 & 1.19555053 \\
\hline & -0.82413401 & -0.59010578 & -1.02042086 \\
\hline & -0.84081992 & 0.48704719 & 1.04304185 \\
\hline & 1.91375615 & 0.13593459 & -1.0153 \\
\hline & 4.25840087 & 2.33924938 & -0.16109446 \\
\hline & 1.74957490 & 0.81809029 & 1.98949005 \\
\hline & 3.27630460 & 0.59400214 & 1.14626717 \\
\hline & 4.26942688 & 4.71712183 & -0.86835 \\
\hline & 2.25863639 & 6.13950216 & -0.49284879 \\
\hline & 0.24260356 & 5.15616553 & 0.59039804 \\
\hline & 0.23581319 & 2.76497180 & 1.27534128 \\
\hline & 2.72967249 & -6.75618000 & 1.51030753 \\
\hline & 0.57425160 & -6.84573154 & -0.22528272 \\
\hline & 4.54499723 & -4.50095884 & 1.7124290 \\
\hline & 3.41293543 & -4.36034465 & 3.08721756 \\
\hline & 3.58776661 & -3.01424364 & 1.93291508 \\
\hline & -2.57118492 & 0.51283131 & -1.3728448 \\
\hline & -2.83187732 & -1.19134345 & -0.9213593 \\
\hline & -2.87894208 & 0.96674268 & 0.9795188 \\
\hline & -2.51094090 & -0.71857607 & 1.42863279 \\
\hline & -1.21948187 & -4.66050888 & -0.7086691 \\
\hline & -0.06833042 & -4.64060101 & -2.0728592 \\
\hline & -0.29905237 & -3.17979684 & -1.08127 \\
\hline
\end{tabular}

\section{IN1'}

$\mathrm{E}=-2508.79682551 \mathrm{au} \quad \mathrm{ZPE}=211.1 \mathrm{kcal} / \mathrm{mol}$

$-0.85211400$

$\begin{array}{lll}-4.35251500 & -0.95189000 & 0.38685800\end{array}$

$\begin{array}{lll}0.47274100 & -1.71021300 & -1.69242900\end{array}$

$1.36877600-0.63823600 \quad-1.55248800$

$-3.77206100 \quad-1.84542300 \quad 1.23310300$

$\begin{array}{lll}2.59512000 & -0.67317700 & -0.70678300 \\ 3.12445200 & -1.87689600 & -0.20107400\end{array}$

$3.12445200-1.87689600 \quad-0.20107400$

$\begin{array}{lll}4.29655700 & -1.88406800 & 0.56312100\end{array}$

$\begin{array}{lll}4.97343300 & -0.68964000 & 0.83055100\end{array}$

$\begin{array}{lll}4.46351200 & 0.51474400 & 0.32693700\end{array}$

$\begin{array}{lll}3.28921100 & 0.52147000 & -0.42638700\end{array}$

$\begin{array}{lll}-3.50080400 & 0.69322000 & -1.35184400\end{array}$

$\begin{array}{lll}-0.34712800 & -0.23840800 & -0.56647800\end{array}$

$\begin{array}{lll}-3.31688000 & -0.35166900 & -0.33898500\end{array}$

$\begin{array}{lll}-2.39254000 & -1.77845200 & 0.99747300\end{array}$

$\begin{array}{lll}-1.75592000 & -3.56234900 & 1.90797300\end{array}$

$\begin{array}{lll}-0.48212100 & -2.59553600 & 1.12760100\end{array}$

$\begin{array}{lll}-1.15360000 & -2.07394200 & 2.68858800\end{array}$

$\begin{array}{lll}-5.38664700 & -0.69446100 & 0.23560400\end{array}$

$\begin{array}{lll}-0.10117900 & -1.81570200 & -2.61110600\end{array}$

$\begin{array}{lll}1.40805900 & 0.09327400 & -2.35429100\end{array}$

$\begin{array}{lll}-4.20308300 & -2.50572500 & 1.96582400\end{array}$

$\begin{array}{lll}2.63574100 & -2.81760100 & -0.43215400\end{array}$

$\begin{array}{lll}4.68748800 & -2.82605900 & 0.93729000\end{array}$

$\begin{array}{lll}5.88537400 & -0.69550600 & 1.41967600\end{array}$

$\begin{array}{lll}4.97704200 & 1.44955400 & 0.53097700\end{array}$

$\begin{array}{lll}2.87755800 & 1.45981600 & -0.78226800\end{array}$

$\begin{array}{lll}-4.50503300 & 1.11062100 & -1.24963400\end{array}$

$\begin{array}{lll}-3.38994800 & 0.27794900 & -2.35828200\end{array}$

$-2.76117700-1.48339300-1.19551000$

$\begin{array}{lll}-0.18393100 & 1.66873800 & 0.07593700\end{array}$

$\begin{array}{lll}-0.97682400 & 2.69642400 & -0.45769100\end{array}$

$0.61736300 \quad 2.16662600 \quad 1.09409700$

$\begin{array}{lll}-0.66636200 & 3.98557700 & 0.21422200\end{array}$

$0.36017800 \quad 3.61364100 \quad 1.29883800$

$\begin{array}{lll}-0.26797300 & 4.67521300 & -0.53506700\end{array}$

$\begin{array}{lll}-1.59462500 & 4.39543600 & 0.62070800\end{array}$

$\begin{array}{lll}1.30752500 & 4.14853900 & 1.19601200\end{array}$

$\begin{array}{lll}-0.02060900 & 3.75385600 & 2.31426300\end{array}$

$0.62405800-2.63824400-1.14495700$

\section{TS1'}

$\begin{array}{crrr}\text { E }=-2508.76594660 \mathrm{au} & \mathrm{ZPE}=211.8 \mathrm{kcal} / \mathrm{mol} \\ \mathrm{Cu} & 0.72178100 & -0.87805500 & -0.32226300 \\ \mathrm{C} & -1.18987300 & -0.96549300 & -0.99825100 \\ \mathrm{~B} & -0.40761300 & 0.78615000 & -0.58894600 \\ \mathrm{C} & -0.50473800 & -2.30888200 & -0.83209000 \\ \mathrm{C} & 1.86280900 & 0.48090100 & 2.54883300 \\ \mathrm{O} & -0.41294800 & 1.59964900 & -1.71384000 \\ \mathrm{O} & -0.68991000 & 1.51607400 & 0.56527700 \\ \mathrm{C} & 2.41280300 & -0.32539000 & 0.24242500 \\ \mathrm{~N} & 2.79378000 & 0.22252700 & 1.44895200 \\ \mathrm{~N} & 3.59347900 & -0.40473400 & -0.46702500 \\ \mathrm{C} & 4.17259100 & 0.46036900 & 1.48795300 \\ \mathrm{C} & 4.67393400 & 0.07438300 & 0.28278100 \\ \mathrm{C} & -0.63497000 & 3.00122400 & -1.29753300 \\ \mathrm{C} & -1.07092200 & 2.89525100 & 0.17671000 \\ \mathrm{C} & 3.67245100 & -0.92591000 & -1.82941000 \\ \mathrm{H} & 4.66161800 & 0.87280700 & 2.35358400 \\ \mathrm{H} & 5.67860400 & 0.09903500 & -0.10260000 \\ \mathrm{H} & -2.15186300 & 2.99848800 & 0.30566800 \\ \mathrm{H} & -0.55541100 & 3.59750600 & 0.83473000 \\ \mathrm{H} & -1.39861700 & 3.43723700 & -1.94444500 \\ \mathrm{H} & 0.30275300 & 3.55114300 & -1.42168800 \\ \mathrm{H} & 2.90874900 & -1.70009100 & -1.94380000 \\ \mathrm{H} & 3.49186200 & -0.13703900 & -2.56639300 \\ \mathrm{H} & \mathbf{4 . 6 5 9 4 4 2 0 0} & -1.36251100 & -1.99951300 \\ \mathrm{H} & 0.91823400 & 0.84645400 & 2.13889200\end{array}$




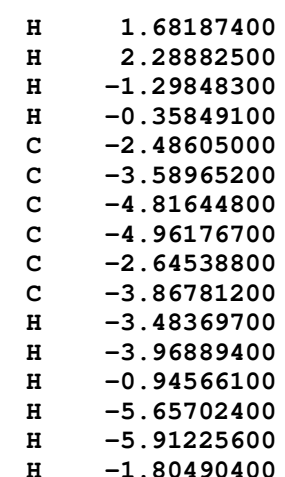

-0.43155600
1.24077500
-0.70012500
-2.83940400
-0.75015800
-0.18633800
-0.01200300
-0.39123800
-1.12009500
-0.94567200
0.10585500
-1.24134600
-2.94930400
0.41459900
-0.25910400
-1.54660100

3.12708900
3.20830100
-2.04810700
-1.77280300
-0.25913300
-0.92635600
-0.27468600
1.06367500
1.09034600
1.74220700
-1.96752800
2.78260000
-0.06874900
-0.81478800
1.57160400
1.62849000

\section{A'}

$\mathrm{E}=-2508.63023939 \mathrm{au} \quad \mathrm{ZPE}=211.7 \mathrm{kcal} / \mathrm{mol}$

\begin{tabular}{|c|c|c|c|}
\hline & -0.84556400 & 0.71083900 & 0.27738500 \\
\hline C & 2.70274200 & -0.38353700 & -0.26943100 \\
\hline & 3.57721800 & -2.01523100 & 1.39846700 \\
\hline & 4.63023900 & 0.78389400 & -0.64987500 \\
\hline & -0.86079700 & -1.66157400 & -0.35475400 \\
\hline & -1.61769800 & -0.31770300 & -0.60958200 \\
\hline & 4.92159500 & -0.17396000 & 0.27185800 \\
\hline & -3.13312900 & -0.28453300 & -0.39521600 \\
\hline & -3.85742900 & -1.41034700 & 0.02302800 \\
\hline & -5.24616200 & -1.35044600 & 0.19769300 \\
\hline & -5.93901400 & -0.15956900 & -0.04287100 \\
\hline & -5.22989000 & 0.97373000 & -0.46245600 \\
\hline & -3.84483400 & 0.90787600 & -0.63447400 \\
\hline & -0.17183000 & 1.88499700 & $2.17 \epsilon$ \\
\hline & 0.45655200 & 2.55633200 & 0.92817400 \\
\hline & 2.57608100 & 1.45886300 & -1.97070300 \\
\hline & 0.95624800 & -1.07222400 & -0.33482000 \\
\hline & 3.28334800 & 0.65056000 & -0.96815100 \\
\hline & 3.73560400 & -0.87810500 & 0.49163400 \\
\hline & -0.80346000 & 0.69379100 & 1.68411900 \\
\hline & -0.16745100 & 1.85280600 & -0.21324100 \\
\hline & 3.75501000 & -1.71523800 & 2.43452600 \\
\hline & 2.54678300 & -2.36003500 & 1.29375900 \\
\hline & 4.2636 & -2.822 & 1.12847900 \\
\hline & 5.25481800 & 1.54148300 & -1.10035300 \\
\hline & -1.15686300 & -2.05104800 & 0.63076700 \\
\hline & 5.84054900 & -0.40945200 & 0.78102500 \\
\hline & -3.32784600 & -2.33805800 & 0.20922900 \\
\hline & -5.78585400 & -2.23624300 & 0.52130700 \\
\hline & -7.01551800 & -0.11343300 & 0.09279300 \\
\hline & -5.75594000 & 1.90463800 & -0.65566000 \\
\hline & -3.30072800 & 1.78807200 & -0.96657400 \\
\hline & 0.57685500 & 1.61299100 & 2.92764900 \\
\hline & -0.93405700 & 2.51909300 & 2.64616700 \\
\hline & 0.23161700 & 3.62103800 & 0.84405100 \\
\hline & 1.53899800 & 2.40306500 & 0.87463600 \\
\hline & 2.67583300 & 1.01240500 & -2.96454800 \\
\hline & 2. 99990100 & 2.46594200 & -1.98539300 \\
\hline & 1.52206300 & 1.51874200 & -1.69429300 \\
\hline & -1.14241500 & -2.41212000 & -1.1077080 \\
\hline & -1.43748300 & -0.02131500 & -1.6557160 \\
\hline
\end{tabular}

\section{$\mathrm{CH}_{2} \mathbf{C H C N}$}

$\begin{array}{crrr}\mathrm{E}=-170.817436856 \mathrm{au} \quad \mathrm{ZPE}=31.7 \mathrm{kcal} / \mathrm{mol} \\ \mathrm{C} & 1.31691400 & 0.99362000 & 0.00000000 \\ \mathrm{C} & 0.00000000 & 0.77569800 & 0.00000000 \\ \mathrm{C} & -0.57945000 & -0.53224000 & 0.00000000 \\ \mathrm{~N} & -1.06522800 & -1.60190500 & 0.00000000 \\ \mathrm{H} & 2.03124900 & 0.17960100 & 0.00000000 \\ \mathrm{H} & 1.70728700 & 2.00716900 & 0.00000000 \\ \mathrm{H} & -0.70672100 & 1.60409400 & 0.00000000\end{array}$

IN1 ${ }_{\mathrm{CN}}$

$\mathrm{E}=-2370.01739873 \mathrm{au} \quad \mathrm{ZPE}=159.1 \mathrm{kcal} / \mathrm{mol}$

$\begin{array}{lrrr}\mathrm{Cu} & -0.00753400 & -0.70262100 & -0.34051700 \\ \mathrm{C} & 0.29167700 & -2.53930500 & -0.90398500 \\ \mathrm{~B} & 1.87145900 & -0.28112200 & 0.19240600 \\ \mathrm{C} & -1.08083900 & -2.24290500 & -1.16221600 \\ \mathrm{C} & 0.02791200 & 2.27375400 & -1.86362100 \\ \mathrm{O} & 2.52859500 & -0.69806700 & 1.33439600 \\ \mathrm{O} & 2.67762200 & 0.56148800 & -0.57245400 \\ \mathrm{C} & -1.04274100 & 0.90512600 & -0.05721700 \\ \mathrm{~N} & -0.89886100 & 2.08821700 & -0.74143700 \\ \mathrm{~N} & -1.98063000 & 1.19203700 & 0.90382800 \\ \mathrm{C} & -1.73160000 & 3.08429900 & -0.22042200 \\ \mathrm{C} & -2.40792900 & 2.52155000 & 0.81843200 \\ \mathrm{C} & 3.88736900 & -0.09982400 & 1.38498900 \\ \mathrm{C} & 4.00990400 & 0.70339000 & 0.07585800 \\ \mathrm{C} & -2.43597500 & 0.23880900 & 1.92471800 \\ \mathrm{H} & -1.76731800 & 4.07893600 & -0.62965400 \\ \mathrm{H} & -3.13990300 & 2.93515400 & 1.49011300 \\ \mathrm{H} & 4.76409000 & 0.30182200 & -0.60551800 \\ \mathrm{H} & 4.20034300 & 1.76691500 & 0.23805700 \\ \mathrm{H} & 4.61385200 & -0.91189400 & 1.46107800 \\ \mathrm{H} & 3.95180400 & 0.52545400 & 2.27906100 \\ \mathrm{H} & -3.29651000 & 0.66468300 & 2.44368400 \\ \mathrm{H} & -2.73401700 & -0.70283800 & 1.46195100 \\ \mathrm{H} & -1.63876100 & 0.05084000 & 2.64988800 \\ \mathrm{H} & 0.99724500 & 1.83521700 & -1.61199100 \\ \mathrm{H} & -0.36543500 & 1.80263900 & -2.76942700 \\ \mathrm{H} & 0.15123200 & 3.34317000 & -2.04638900 \\ \mathrm{H} & 0.96971000 & -2.61184300 & -1.75130800 \\ \mathrm{H} & -1.39618900 & -1.99417800 & -2.16940700 \\ \mathrm{H} & 0.55082400 & -3.17962000 & -0.06351900 \\ \mathrm{C} & -2.12036400 & -2.68249900 & -0.29859400 \\ \mathrm{~N} & -2.98250600 & -2.99519500 & 0.44520000\end{array}$

\section{TS1 $_{\mathbf{C N}}$}

$\mathrm{E}=-2370.00110931 \mathrm{au} \quad \mathrm{ZPE}=158.6 \mathrm{kcal} / \mathrm{mol}$

$\begin{array}{lrrr}\mathrm{Cu} & -0.02505700 & 0.53377000 & -0.42515300 \\ \mathrm{C} & -1.62942800 & 1.67506800 & -0.61374900 \\ \mathrm{~B} & -1.86674500 & -0.08528900 & 0.11786300 \\ \mathrm{C} & -0.39302200 & 2.37305700 & -1.13541200 \\ \mathrm{C} & 0.72400700 & -2.65346900 & -1.34278600 \\ \mathrm{O} & -2.34915800 & -0.10110200 & 1.41382400 \\ \mathrm{O} & -2.46281900 & -1.07043400 & -0.66705600 \\ \mathrm{C} & 1.42492800 & -0.59981800 & -0.08379900 \\ \mathrm{~N} & 1.64641700 & -1.89333300 & -0.49731100 \\ \mathrm{~N} & 2.54846400 & -0.28775800 & 0.64690300 \\ \mathrm{C} & 2.87959900 & -2.36859700 & -0.03864800 \\ \mathrm{C} & 3.44164700 & -1.36216500 & 0.68630500 \\ \mathrm{C} & -3.30057900 & -1.22218500 & 1.57028700 \\ \mathrm{C} & -3.49098500 & -1.77260500 & 0.13985500 \\ \mathrm{C} & 2.76976600 & 1.00392000 & 1.30857800 \\ \mathrm{H} & 3.23951700 & -3.35643700 & -0.26909300 \\ \mathrm{H} & 4.37744500 & -1.31403400 & 1.21579300 \\ \mathrm{H} & -4.47209300 & -1.53564200 & -0.28008100 \\ \mathrm{H} & -3.31633000 & -2.84776600 & 0.06395400 \\ \mathrm{H} & -4.22179400 & -0.83219700 & 2.00768100 \\ \mathrm{H} & -2.85937400 & -1.95442900 & 2.25180900 \\ \mathrm{H} & 2.16755700 & 1.77216600 & 0.82109900 \\ \mathrm{H} & 2.49122500 & 0.95108800 & 2.36540800 \\ \mathrm{H} & 3.82355900 & 1.27926000 & 1.22490100 \\ \mathrm{H} & -0.27852400 & -2.23418200 & -1.23795200 \\ \mathrm{H} & 1.02702800 & -2.60199300 & -2.39356600 \\ \mathrm{H} & 0.71277100 & -3.69904400 & -1.02415100 \\ \mathrm{H} & -2.36782400 & 1.51082600 & -1.39671100 \\ \mathrm{H} & -2.07472300 & 2.16084600 & 0.25576500 \\ \mathrm{C} & 0.21512200 & 3.37501200 & -0.34536800 \\ \mathrm{H} & -0.33217900 & 2.51829300 & -2.21144500 \\ \mathrm{~N} & 0.77563300 & 4.15442600 & 0.35054900\end{array}$

$2 \mathbf{A}_{\mathrm{CN}}$

$\mathrm{E}=-2370.04966475 \mathrm{au} \quad \mathrm{ZPE}=159.8 \mathrm{kcal} / \mathrm{mol}$

B $\quad 2.91871200 \quad 0.13567200 \quad-0.24277400$ 


$\begin{array}{lr}\mathrm{C} & -1.97615700 \\ \mathrm{C} & -3.62965300 \\ \mathrm{C} & -3.43340800 \\ \mathrm{C} & 1.04538600 \\ \mathrm{C} & 2.25153600 \\ \mathrm{C} & -4.16769100 \\ \mathrm{C} & 4.59218400 \\ \mathrm{C} & 3.21459900 \\ \mathrm{C} & -0.96205500 \\ \mathrm{Cu} & -0.43881800 \\ \mathrm{~N} & -2.10048500 \\ \mathrm{~N} & -3.26698600 \\ \mathrm{O} & 4.28205300 \\ \mathrm{O} & 2.23764200 \\ \mathrm{H} & -4.28397200 \\ \mathrm{H} & -2.71149400 \\ \mathrm{H} & -4.13279200 \\ \mathrm{H} & -3.73418300 \\ \mathrm{H} & 1.37705300 \\ \mathrm{H} & 3.02659200 \\ \mathrm{H} & 1.91413400 \\ \mathrm{H} & -5.22598600 \\ \mathrm{H} & 5.25428800 \\ \mathrm{H} & 5.10886700 \\ \mathrm{H} & 2.98771400 \\ \mathrm{H} & 3.10411800 \\ \mathrm{H} & -0.92075100 \\ \mathrm{H} & -1.04014500 \\ \mathrm{H} & -0.05321200 \\ \mathrm{~N} & -0.17348100 \\ \mathrm{C} & 0.41376400 \\ & \end{array}$

0.03822600
-0.11826400
-0.18833900
0.42608600
-0.49654700
-0.24491500
-0.05058200
0.18314300
0.09232100
0.25113500
-0.01643600
-0.10610400
-0.28120100
0.01684100
0.72555500
-0.03571900
-1.05437700
-0.25546400
1.47487500
-0.44349700
-1.54532200
-0.37129000
0.81511800
-0.93125600
-0.54886400
1.19086500
-0.77952300
1.00100100
0.13259900
-0.14938900
0.12131500

IN1 $_{\mathrm{CN}^{\prime}}$

$\mathrm{E}=-2370.00802040 \mathrm{au} \mathrm{ZPE}=158.7 \mathrm{kcal} / \mathrm{mol}$

$\begin{array}{lrrr}\mathrm{Cu} & 0.21479200 & -0.65528600 & -0.41143100 \\ \mathrm{C} & 1.53309100 & -1.95266400 & -1.10989400 \\ \mathrm{~B} & 1.30237200 & 0.95136600 & 0.12245400 \\ \mathrm{C} & 0.22126100 & -2.48925700 & -1.22913600 \\ \mathrm{C} & -1.79527200 & 1.67104000 & -1.77695000 \\ \mathrm{O} & 1.95804300 & 1.14345700 & 1.32318500 \\ \mathrm{O} & 1.41703200 & 2.07829000 & -0.69543300 \\ \mathrm{C} & -1.59675700 & -0.12527800 & -0.04251500 \\ \mathrm{~N} & -2.30404000 & 0.88484700 & -0.64797100 \\ \mathrm{~N} & -2.43412900 & -0.56235900 & 0.95565300 \\ \mathrm{C} & -3.55238200 & 1.06768800 & -0.04148000 \\ \mathrm{C} & -3.63241100 & 0.16264600 & 0.97168000 \\ \mathrm{C} & 2.60667200 & 2.47932500 & 1.34407600 \\ \mathrm{C} & 2.25844200 & 3.10401100 & -0.02047500 \\ \mathrm{C} & -2.07976900 & -1.60835500 & 1.91543100 \\ \mathrm{H} & -0.08927700 & -3.28355200 & -0.55421700 \\ \mathrm{H} & -4.25559500 & 1.80769700 & -0.38210500 \\ \mathrm{H} & -4.41284400 & -0.02715100 & 1.68805900 \\ \mathrm{H} & 3.13597300 & 3.28809500 & -0.64498700 \\ \mathrm{H} & 1.67589500 & 4.02503100 & 0.06262000 \\ \mathrm{H} & 3.67941800 & 2.33338500 & 1.48861100 \\ \mathrm{H} & 2.20273400 & 3.04168600 & 2.18972200 \\ \mathrm{H} & -2.98961800 & -2.08232700 & 2.28962700 \\ \mathrm{H} & -1.46754500 & -2.35818100 & 1.41228600 \\ \mathrm{H} & -1.51447700 & -1.19253000 & 2.75495000 \\ \mathrm{H} & -0.75222300 & 1.94255100 & -1.59143300 \\ \mathrm{H} & -1.87017400 & 1.10319400 & -2.70952000 \\ \mathrm{H} & -2.39118600 & 2.58108200 & -1.86974800 \\ \mathrm{H} & 2.02222400 & -1.53350300 & -1.98850400 \\ \mathrm{H} & -0.25184000 & -2.49833700 & -2.20836100 \\ \mathrm{C} & 2.43997600 & -2.44988300 & -0.11691000 \\ \mathrm{~N} & 3.18388300 & -2.86967300 & 0.69192700\end{array}$

TS1 $_{\mathrm{CN}^{\prime}}$

$\begin{array}{ccrr}\mathrm{E}= & -2369.97855074 \mathrm{au} \quad \mathrm{ZPE}=158.3 \mathrm{kcal} / \mathrm{mol} \\ \mathrm{Cu} & 0.06851900 & -0.84030400 & -0.56906300 \\ \mathrm{C} & 2.04862200 & -0.94572500 & -0.88533200 \\ \mathrm{~B} & 1.28498000 & 0.65343300 & 0.02766200 \\ \mathrm{C} & 1.25417300 & -2.11649400 & -1.45650300 \\ \mathrm{C} & -2.04589400 & 1.44316300 & -1.83816900\end{array}$

$\begin{array}{rr}\mathrm{O} & 1.56252200 \\ \mathrm{O} & 1.36705400 \\ \mathrm{C} & -1.64480700 \\ \mathrm{~N} & -2.44357700 \\ \mathrm{~N} & -2.40892500 \\ \mathrm{C} & -3.67778600 \\ \mathrm{C} & -3.65368700 \\ \mathrm{C} & 1.96595800 \\ \mathrm{C} & 1.70048500 \\ \mathrm{C} & -1.93947600 \\ \mathrm{H} & -4.44554000 \\ \mathrm{H} & -4.39133100 \\ \mathrm{H} & 2.57341700 \\ \mathrm{H} & 0.85034600 \\ \mathrm{H} & 3.02090900 \\ \mathrm{H} & 1.36639300 \\ \mathrm{H} & -1.34435100 \\ \mathrm{H} & -1.31916900 \\ \mathrm{H} & -2.79732300 \\ \mathrm{H} & -0.99984800 \\ \mathrm{H} & -2.16507500 \\ \mathrm{H} & -2.67105100 \\ \mathrm{H} & 2.48958100 \\ \mathrm{C} & 3.04053800 \\ \mathrm{H} & 1.28721100 \\ \mathrm{~N} & 3.84237900 \\ \mathrm{H} & 1.47937300 \\ & \end{array}$

0.88141800
1.81376200
-0.28738100
0.66852200
-0.76733200
0.76759100
-0.12910200
2.29563200
2.94955000
-1.79180900
1.45066500
-0.36512500
3.46119900
3.63679600
2.31126500
2.72603500
-2.52325600
-1.35076700
-2.29433500
1.74233500
0.85030400
2.33578200
-0.30991300
-1.31143400
-2.16697600
-1.63485800
-3.07206200

1. 35656000

$-0.73586000$

$-0.07534600$

$-0.66113400$

0.96374800

$-0.00736600$

1. 01644400

1.53406700

0.15927500

1.89633200

$-0.32718400$

1. 76365800

$-0.25059900$

0.17280600

1. 81755800

2. 33847200

1. 34367600

2. 68220000

2. 34778500

$-1.73815200$

$-2.75083900$

$-1.90767500$

$-1.65153300$

0.10265800

$-2.54366500$

0.89920100

$-0.98665800$

\section{$2 \mathbf{A}_{\mathbf{C N}}{ }^{\prime}$}

$\mathrm{E}=-2370.03589080 \mathrm{au} \quad \mathrm{ZPE}=159.8 \mathrm{kcal} / \mathrm{mol}$

$\begin{array}{lrrr}\mathrm{B} & -2.40066600 & 0.46961200 & -0.01986000 \\ \mathrm{C} & 2.14468700 & -0.14837700 & -0.06157400 \\ \mathrm{C} & 3.40384200 & -1.86247900 & 1.25206700 \\ \mathrm{C} & 3.89885900 & 1.24547000 & -0.55518400 \\ \mathrm{C} & -1.29216700 & -1.80583600 & 0.04648000 \\ \mathrm{C} & -2.35214500 & -0.93394200 & -0.71622800 \\ \mathrm{C} & 4.39824600 & 0.21596000 & 0.18410100 \\ \mathrm{C} & -3.67532500 & -1.56269600 & -0.79233000 \\ \mathrm{C} & -2.85743400 & 2.13628700 & 1.52886900 \\ \mathrm{C} & -1.92175000 & 2.70205300 & 0.43107700 \\ \mathrm{C} & 1.60847400 & 1.86811900 & -1.45030900 \\ \mathrm{Cu} & 0.43902500 & -0.95294700 & 0.01824300 \\ \mathrm{~N} & 2.52704200 & 1.01094400 & -0.69540600 \\ \mathrm{~N} & 3.31947400 & -0.62418200 & 0.47613000 \\ \mathrm{O} & -3.08040300 & 0.73392000 & 1.13970500 \\ \mathrm{O} & -1.71318800 & 1.57332100 & -0.49922500 \\ \mathrm{H} & 3.72389000 & -1.65659400 & 2.27769900 \\ \mathrm{H} & 2.40684300 & -2.30500300 & 1.26654300 \\ \mathrm{H} & 4.10300200 & -2.56192800 & 0.78495500 \\ \mathrm{H} & 4.38643900 & 2.10382800 & -0.98465500 \\ \mathrm{H} & -1.67583200 & -1.98001800 & 1.06201800 \\ \mathrm{H} & 5.40040100 & 0.01316000 & 0.52103900 \\ \mathrm{H} & -2.40543100 & 2.15008900 & 2.52313400 \\ \mathrm{H} & -3.82560500 & 2.64025200 & 1.56508500 \\ \mathrm{H} & -2.36754100 & 3.52775000 & -0.12795600 \\ \mathrm{H} & -0.94872700 & 3.01219300 & 0.82085700 \\ \mathrm{H} & 1.80934100 & 1.79465500 & -2.52360300 \\ \mathrm{H} & 1.72275600 & 2.90896800 & -1.13382300 \\ \mathrm{H} & 0.58579200 & 1.54332500 & -1.25229100 \\ \mathrm{H} & -1.26477900 & -2.79382200 & -0.43800000 \\ \mathrm{H} & -2.01125700 & -0.78032100 & -1.74572900 \\ \mathrm{~N} & -4.72518600 & -2.08882300 & -0.83367600\end{array}$

\section{$\mathrm{CH}_{2} \mathrm{CHMe}$}

$\mathrm{E}=-117.917358927 \mathrm{au} \quad \mathrm{ZPE}=50.2 \mathrm{kcal} / \mathrm{mol}$

$\begin{array}{rrrr}\text { C } & -1.23759200 & 0.16231000 & 0.00007600 \\ \mathrm{C} & 0.13690200 & -0.45453200 & -0.00028700 \\ \mathrm{C} & 1.28234500 & 0.22086800 & 0.00008900 \\ \mathrm{H} & -1.18230500 & 1.25582100 & -0.00027400 \\ \mathrm{H} & -1.80913900 & -0.15430400 & 0.88243700 \\ \mathrm{H} & -1.81006400 & -0.15439100 & -0.88154700 \\ \mathrm{H} & 0.16993000 & -1.54633100 & 0.00000000 \\ \mathrm{H} & 2.24227400 & -0.28289400 & 0.00045700\end{array}$




\section{H}

-1.13150000
-2.10058400
-0.33061600

3.49703300

.26609700

4.35526000

0.94544100

$-0.31936500$

$-0.37712300$
IN1 $_{\text {Me }}$

$\mathrm{E}=-2317.09759895 \mathrm{au} \quad \mathrm{ZPE}=177.2 \mathrm{kcal} / \mathrm{mol}$

$\begin{array}{lrrr}\mathrm{C} & 1.23133100 & -0.62450700 & -0.05366600 \\ \mathrm{C} & 2.32989400 & -0.11440400 & 2.14171300 \\ \mathrm{C} & 2.14202600 & -2.70917200 & -0.37832000 \\ \mathrm{C} & 0.99766700 & 2.51791500 & -0.90472600 \\ \mathrm{C} & -0.38248200 & 2.72654100 & -0.77594100 \\ \mathrm{C} & 2.65734900 & -2.22350500 & 0.78319300 \\ \mathrm{C} & 2.00510500 & 3.12930300 & 0.05246700 \\ \mathrm{C} & 0.45617700 & -1.86324300 & -2.08402600 \\ \mathrm{Cu} & 0.00160800 & 0.85168400 & -0.24810200 \\ \mathrm{~N} & 1.27533500 & -1.72921800 & -0.87556000 \\ \mathrm{~N} & 2.09899100 & -0.95061600 & 0.96609100 \\ \mathrm{H} & 3.38663600 & -0.14547900 & 2.42051000 \\ \mathrm{H} & 2.05691000 & 0.91166600 & 1.89884300 \\ \mathrm{H} & 1.72164300 & -0.45288000 & 2.98703500 \\ \mathrm{H} & 2.31152700 & -3.64182300 & -0.88834900 \\ \mathrm{H} & 1.38371300 & 2.30849300 & -1.90428900 \\ \mathrm{H} & -1.02647800 & 2.72195900 & -1.65323000 \\ \mathrm{H} & -0.77351700 & 3.28736400 & 0.06779600 \\ \mathrm{H} & 3.35455700 & -2.65526000 & 1.48047100 \\ \mathrm{H} & 0.52756700 & -2.89048900 & -2.44777100 \\ \mathrm{H} & 0.81130300 & -1.18530200 & -2.86580300 \\ \mathrm{H} & -0.58606600 & -1.63148500 & -1.84439500 \\ \mathrm{~B} & -1.81858300 & 0.09657100 & 0.18593800 \\ \mathrm{O} & -2.48833000 & -0.76937200 & -0.69021200 \\ \mathrm{O} & -2.56825700 & 0.28109000 & 1.34172300 \\ \mathrm{C} & -3.79856900 & -1.17001200 & -0.11296600 \\ \mathrm{C} & -3.83169700 & -0.49495100 & 1.26968800 \\ \mathrm{H} & -4.58950600 & -0.81810900 & -0.78092700 \\ \mathrm{H} & -3.83330100 & -2.26150100 & -0.06045800 \\ \mathrm{H} & -4.66752000 & 0.19923100 & 1.38842500 \\ \mathrm{H} & -3.84234300 & -1.21149200 & 2.09581000 \\ \mathrm{H} & 1.57167100 & 3.26696800 & 1.05160800 \\ \mathrm{H} & 2.32251100 & 4.12243000 & -0.29982200 \\ \mathrm{H} & 2.90860000 & 2.51532700 & 0.14951300 \\ & & & \end{array}$

TS1 $_{\text {Me }}$

$\mathrm{E}=-2317.06893519 \mathrm{au} \quad \mathrm{ZPE}=176.7 \mathrm{kcal} / \mathrm{mol}$

$\begin{array}{lr}\text { Cu } & -0.13946900 \\ \text { C } & -2.04957100 \\ \text { B } & -1.64406200 \\ \mathrm{C} & -1.10761500 \\ \mathrm{C} & 1.38269700 \\ \mathrm{O} & -2.05171000 \\ \mathrm{O} & -1.93337300 \\ \mathrm{C} & 1.49986200 \\ \mathrm{~N} & 2.05054000 \\ \mathrm{~N} & 2.46224300 \\ \mathrm{C} & 3.32065000 \\ \mathrm{C} & 3.57618000 \\ \mathrm{C} & -2.58000900 \\ \mathrm{C} & -2.66188700 \\ \mathrm{C} & 2.30613900 \\ \mathrm{H} & 3.91515200 \\ \mathrm{H} & 4.42947900 \\ \mathrm{H} & -3.68914900 \\ \mathrm{H} & -2.17659600 \\ \mathrm{H} & -3.55241000 \\ \mathrm{H} & -1.88893800 \\ \mathrm{H} & 1.71908600 \\ \mathrm{H} & 1.78592600 \\ \mathrm{H} & 3.28806600 \\ \mathrm{H} & 0.31292800 \\ \mathrm{H} & 1.52787400 \\ \mathrm{H} & 1.79531600 \\ \mathrm{H} & -2.65085700 \\ \mathrm{H} & -2.66768000 \\ \mathrm{C} & -1.17272000 \\ \mathrm{H} & -1.11175200\end{array}$

$0.78368800-0.35741200$

$1.29901000-0.54033000$

$-0.46413400 \quad 0.13937400$

$2.40160400-0.94477900$

$-1.75289300-1.92480300$

$-0.70001200 \quad 1.44774500$

$-1.55798500 \quad-0.68322000$

$-0.05341900-0.09094500$

$-1.10465900 \quad-0.79666100$

$0.24209200 \quad 0.85394900$

$-1.43612500 \quad-0.31038000$

$-0.59730900 \quad 0.73031500$

$-2.07440600 \quad 1.55952900$

$-2.57896700 \quad 0.10305200$

$1.30243300 \quad 1.84520300$

$-2.22292100 \quad-0.74198200$

$-0.52397700 \quad 1.38230600$

$-2.63059200-0.26974800$

$-3.54626500-0.04596600$

$-2.03319900 \quad 2.05539400$

$-2.66218600 \quad 2.17110000$

$2.10761200 \quad 1.39531500$

$0.93910200 \quad 2.73728600$

$1.68732500 \quad 2.13090600$

$-1.82751800-1.71308600$

$-1.17928600 \quad-2.84669300$

$-2.75537000-2.06037800$

$0.88894300-1.35188500$

$1.53291000 \quad 0.33091300$

$3.69428200-0.13498600$

$2.57742900-2.02281900$ $\mathbf{2} \mathbf{A}_{\mathrm{Me}}$

$\mathrm{E}=-2317.12152337 \mathrm{au} \quad \mathrm{ZPE}=178.3 \mathrm{kcal} / \mathrm{mol}$

$\begin{array}{lrrr}\mathrm{B} & 2.73113500 & -0.09798900 & -0.21358400 \\ \mathrm{C} & -1.88049600 & -0.16834700 & 0.01208500 \\ \mathrm{C} & -3.37099900 & 1.40689300 & 1.25163900 \\ \mathrm{C} & -3.40429800 & -1.84395200 & -0.36084200 \\ \mathrm{C} & 1.26836800 & 2.00657300 & -0.08313200 \\ \mathrm{C} & 2.37409300 & 1.25462300 & -0.89016800 \\ \mathrm{C} & -4.04846300 & -0.86081400 & 0.32795600 \\ \mathrm{C} & 1.04765300 & 3.41094700 & -0.68622300 \\ \mathrm{C} & 3.70014200 & -1.62862900 & 1.25734000 \\ \mathrm{C} & 2.69690400 & -2.37110900 & 0.34057400 \\ \mathrm{C} & -1.05481800 & -2.15827100 & -1.26652900 \\ \mathrm{Cu} & -0.32137700 & 0.89836100 & 0.00036900 \\ \mathrm{~N} & -2.08724200 & -1.40968100 & -0.54423700 \\ \mathrm{~N} & -3.10921800 & 0.15179400 & 0.54695100 \\ \mathrm{O} & 3.64207100 & -0.22896400 & 0.81444600 \\ \mathrm{O} & 2.15666600 & -1.32336100 & -0.54391700 \\ \mathrm{H} & -3.63488700 & 1.21852900 & 2.29668300 \\ \mathrm{H} & -2.45330000 & 1.99583900 & 1.21038900 \\ \mathrm{H} & -4.17955000 & 1.96054500 & 0.76540200 \\ \mathrm{H} & -3.75799400 & -2.79193800 & -0.72869000 \\ \mathrm{H} & 1.67493400 & 2.15550600 & 0.93224200 \\ \mathrm{H} & 3.28560800 & 1.87548200 & -0.97703600 \\ \mathrm{H} & 2.02264000 & 1.06787600 & -1.91246700 \\ \mathrm{H} & -5.06577200 & -0.79521200 & 0.67423400 \\ \mathrm{H} & 3.42241500 & -1.67136100 & 2.31360100 \\ \mathrm{H} & 4.72616500 & -1.98718700 & 1.14257200 \\ \mathrm{H} & 3.17054700 & -3.13702800 & -0.27898300 \\ \mathrm{H} & 1.86836600 & -2.82028200 & 0.89530400 \\ \mathrm{H} & -1.27934800 & -2.18541400 & -2.33753000 \\ \mathrm{H} & -0.99995900 & -3.18221400 & -0.88487100 \\ \mathrm{H} & -0.09276200 & -1.66620600 & -1.11217900 \\ \mathrm{H} & 0.65130000 & 3.35204500 & -1.71040700 \\ \mathrm{H} & 1.98931400 & 3.98762100 & -0.73868500 \\ \mathrm{H} & 0.33333900 & 3.99721400 & -0.09179600\end{array}$

IN1 $_{\mathrm{Me}^{\prime}}$

$\mathrm{E}=-2317.09929630 \mathrm{au} \quad \mathrm{ZPE}=177.1 \mathrm{kcal} / \mathrm{mol}$

$-0.11326300$

C -1.5564100

$\begin{array}{llrr}\text { C } & -2.69720800 & 0.69976600 & -1.99493600 \\ \text { C } & -3.12623400 & -1.66564300 & 0.71587400\end{array}$

$\begin{array}{llll}\text { C } & -0.21378700 & 2.84400600 & 0.21439000\end{array}$

$\begin{array}{llll}\text { C } & 1.15298100 & 2.56507400 & 0.06802700\end{array}$

$\begin{array}{llll}\text { C } & -3.58124100 & -1.07988200 & -0.42478000\end{array}$

C $\quad-1.04578400 \quad-1.42857200 \quad 2.15263900$

$\mathrm{Cu} \quad 0.11994000 \quad 0.85293800 \quad-0.02573600$

N $\quad-1.89296900 \quad-1.07263600 \quad 1.00981600$

N $\quad-2.61867400 \quad-0.13224500 \quad-0.79655300$

$\mathrm{H} \quad-3.73565000 \quad 0.98745200-2.17815600$

$\mathrm{H} \quad-2.10048300 \quad 1.59758500 \quad-1.83147900$

H $\quad-2.30912400 \quad 0.16777700 \quad-2.86964600$

H $\quad-3.55913600 \quad-2.43278500 \quad 1.33451000$

H $\quad \begin{array}{llll}1.59740200 & 2.71820600 & -0.91216500\end{array}$

H $\quad-4.47950700 \quad-1.24702500-0.99386800$

$\mathrm{H} \quad-1.37157800 \quad-2.39379300 \quad 2.54703200$

H $\quad-1.12804100 \quad-0.67636900 \quad 2.94336000$

H $\quad-0.00516900 \quad-1.50223400 \quad 1.82454000$

B $\quad 1.57144700 \quad-0.51409300-0.32086700$

$\begin{array}{llll}0 & 1.99774300 & -1.40360800 & 0.67615500\end{array}$

O $\quad 2.24698100 \quad-0.77143900-1.50864800$

$\begin{array}{llll}\text { C } & 3.05822800 & -2.30040200 & 0.14567000\end{array}$

$\begin{array}{llll}\text { C } & 3.19031300 & -1.90418400 & -1.33560700\end{array}$

$\mathrm{H} \quad 3.97265400 \quad-2.12673300 \quad 0.71952500$

H $\quad 2.73775700 \quad-3.33588000 \quad 0.28808000$

$\mathrm{H} \quad 4.19301600 \quad-1.55892500 \quad-1.60002400$

H $\quad 2.89576400 \quad-2.70427500 \quad-2.02048300$

$\begin{array}{llll}\text { C } & 2.12743500 & 2.67177800 & 1.22774700\end{array}$

H $2.98248000 \quad 2.00075100 \quad 1.10133400$

$\mathrm{H} \quad 2.50913400 \quad 3.70055600 \quad 1.30702800$ 


$\begin{array}{lr}\mathrm{H} & 1.64330900 \\ \mathrm{H} & -0.78309200 \\ \mathrm{H} & -0.62108200\end{array}$

2.42641300
3.24547400
3.07152900

2.18049300

$-0.62284400$

1.19940800
$\mathrm{H} \quad-4.14236400$
$-4.46234800$
$-1.16351500$

-2.15521300
-1.40613100
-2.92934600

0.00428700

$-1.57297200$

$-0.57067100$
TS1 $_{\mathrm{Me}^{\prime}}$

\begin{tabular}{|c|c|c|c|}
\hline $\mathrm{E}=$ & 317.069953 & au $\quad \mathrm{ZPE}=1$ & $.0 \mathrm{kcal} / \mathrm{mol}$ \\
\hline C & -1.58438200 & -0.08709200 & -0.13310600 \\
\hline C & -2.60116900 & -1.25259500 & 1.82840800 \\
\hline $\mathrm{C}$ & -3.35721900 & 1.26927600 & -0.67065300 \\
\hline C & 1.01783400 & -2.67450300 & -0.31448400 \\
\hline C & 1.99376300 & -1.54605300 & -0.10183000 \\
\hline C & -3.72504900 & 0.53936800 & 0.41781200 \\
\hline C & -1.26909100 & 1.41958900 & -2.10937600 \\
\hline $\mathrm{Cu}$ & 0.05335800 & -0.97821600 & -0.13867600 \\
\hline $\mathbf{N}$ & -2.04898600 & 0.88876700 & -0.99100700 \\
\hline $\mathbf{N}$ & -2.63921600 & -0.28465500 & 0.73502200 \\
\hline $\mathrm{H}$ & -3.60560100 & -1.64291300 & $2.0101250 c$ \\
\hline $\mathrm{H}$ & -1.93978500 & -2.07114900 & $1.5305250 \mathrm{C}$ \\
\hline $\mathrm{H}$ & -2.21595400 & -0.79950900 & 2.74732000 \\
\hline $\mathrm{H}$ & -3.89687200 & 2.00929600 & -1.23612500 \\
\hline $\mathrm{H}$ & 2.49710400 & -1.61850600 & 0.86227000 \\
\hline $\mathrm{H}$ & -4.63969800 & 0.53409300 & 0.98522200 \\
\hline $\mathrm{H}$ & -1.64074600 & 2.41427500 & -2.36717500 \\
\hline $\mathrm{H}$ & -1.35281600 & 0.76949800 & -2.98691400 \\
\hline $\mathrm{H}$ & -0.21980500 & 1.49148800 & -1.81228800 \\
\hline B & 1.53193800 & 0.31400500 & 0.31273500 \\
\hline 0 & 1.86375800 & 1.35003900 & -0.56459400 \\
\hline 0 & 1.81092500 & 0.66911100 & 1.6292640 \\
\hline C & 2.49720300 & 2.45113400 & 0.2000530 \\
\hline C & 2.25375100 & 2.07684700 & 1.67542600 \\
\hline $\mathrm{H}$ & 3.55958100 & 2.48146900 & -0.0592380 \\
\hline $\mathrm{H}$ & 2.02779900 & 3. 39296100 & -0.0915070 \\
\hline $\mathrm{H}$ & 3.15407600 & 2.13760900 & 2.2904460 \\
\hline H & 1.46139800 & 2.67502300 & 2.1355340 \\
\hline C & 2.98583200 & -1.34228900 & -1.2585420 \\
\hline H & 3.63869700 & -0.47342600 & -1.1265040 \\
\hline $\mathrm{H}$ & 3.60927300 & -2.24117700 & -1.34727300 \\
\hline H & 2.45184900 & -1.21238000 & -2.2072010 \\
\hline $\mathrm{H}$ & 0.96903600 & -3.40138800 & 0.4965280 \\
\hline $\mathrm{H}$ & 1.08648500 & -3.15109400 & -1.2935410 \\
\hline
\end{tabular}

$2 \mathbf{A}_{M \mathbf{e}^{\prime}}$

$\mathrm{E}=-2317.12356845 \mathrm{au} \quad \mathrm{ZPE}=178.3 \mathrm{kcal} / \mathrm{mol}$

$\begin{array}{lrrr}\mathrm{B} & -2.56860900 & 0.20522700 & -0.11052600 \\ \mathrm{C} & 2.02469000 & -0.05522600 & -0.03962100 \\ \mathrm{C} & 3.40436000 & -1.72399500 & 1.20596900 \\ \mathrm{C} & 3.66855300 & 1.49321600 & -0.45204900 \\ \mathrm{C} & -1.28483700 & -1.95348100 & -0.06772000 \\ \mathrm{C} & -2.38734300 & -1.16043300 & -0.83954600 \\ \mathrm{C} & 4.24307900 & 0.47269200 & 0.24396900 \\ \mathrm{C} & -3.27421000 & 1.77658300 & 1.46381800 \\ \mathrm{C} & -2.27546000 & 2.44757900 & 0.48927200 \\ \mathrm{C} & 1.33977900 & 1.97548200 & -1.33746200 \\ \mathrm{Cu} & 0.38594900 & -0.99045600 & -0.01754300 \\ \mathrm{~N} & 2.32006200 & 1.15916000 & -0.61573100 \\ \mathrm{~N} & 3.23142100 & -0.46172300 & 0.48688000 \\ \mathrm{O} & -3.39231500 & 0.39456500 & 0.98059600 \\ \mathrm{O} & -1.90467700 & 1.38044000 & -0.45725500 \\ \mathrm{H} & 3.69693900 & -1.54301500 & 2.24468900 \\ \mathrm{H} & 2.44328800 & -2.24029000 & 1.18525600 \\ \mathrm{H} & 4.16033800 & -2.34559700 & 0.71721100 \\ \mathrm{H} & 4.08980000 & 2.40626000 & -0.83675500 \\ \mathrm{H} & -1.67736300 & -2.19180000 & 0.93513800 \\ \mathrm{H} & -2.00325800 & -0.94275800 & -1.84514900 \\ \mathrm{H} & 5.25616300 & 0.33361100 & 0.58077700 \\ \mathrm{H} & -2.91121200 & 1.75503200 & 2.49464100 \\ \mathrm{H} & -4.26445600 & 2.23860800 & 1.44291200 \\ \mathrm{H} & -2.71843800 & 3.27205900 & -0.07550100 \\ \mathrm{H} & -1.36911200 & 2.80082400 & 0.98907400 \\ \mathrm{H} & 1.54879300 & 1.96557200 & -2.41189100 \\ \mathrm{H} & 1.37296900 & 3.00708700 & -0.97447900 \\ \mathrm{H} & 0.34409800 & 1.56506500 & -1.15946000 \\ \mathrm{C} & -3.71046300 & -1.94949800 & -0.98249500 \\ \mathrm{H} & -3.52315400 & -2.91247400 & -1.47531300\end{array}$

\section{$\mathrm{CH}_{2} \mathrm{CHNH}_{2}$}

$\mathrm{E}=-133.952280114 \mathrm{au} \quad \mathrm{ZPE}=43.0 \mathrm{kcal} / \mathrm{mol}$

$\begin{array}{rrrr}\mathrm{C} & 1.27413300 & 0.01298700 & 0.00000000 \\ \mathrm{C} & 0.00000000 & 0.42756600 & 0.00000000 \\ \mathrm{~N} & -1.13268800 & -0.35907100 & 0.00000000 \\ \mathrm{H} & -2.04921500 & 0.05080800 & 0.00000000 \\ \mathrm{H} & -1.06405800 & -1.36338400 & 0.00000000 \\ \mathrm{H} & -0.22115400 & 1.49483000 & 0.00000000 \\ \mathrm{H} & 1.53724100 & -1.04310400 & 0.00000000 \\ \mathrm{H} & 2.08120900 & 0.73102600 & 0.00000000\end{array}$

IN1 NH2 $_{2}$

$\mathrm{E}=-2333.12282488 \mathrm{au} \quad \mathrm{ZPE}=169.6 \mathrm{kcal} / \mathrm{mol}$

$\begin{array}{lrrr}\mathrm{Cu} & -0.05258900 & 0.88968700 & 0.18206600 \\ \mathrm{C} & -0.41451100 & 2.85600300 & 0.21302600 \\ \mathrm{~B} & -1.84568000 & -0.03439600 & 0.16828900 \\ \mathrm{C} & 0.96510900 & 2.67279100 & 0.08197800 \\ \mathrm{C} & 0.80604500 & -1.03213900 & -2.32363900 \\ \mathrm{O} & -2.73498300 & -0.19579500 & 1.22598900 \\ \mathrm{O} & -2.33743200 & -0.68227600 & -0.97485300 \\ \mathrm{C} & 1.28500400 & -0.50132200 & 0.06688700 \\ \mathrm{~N} & 1.50000400 & -1.27308100 & -1.05271200 \\ \mathrm{~N} & 2.06881600 & -1.10397400 & 1.02659700 \\ \mathrm{C} & 2.37971700 & -2.32917200 & -0.79169800 \\ \mathrm{C} & 2.73686600 & -2.22739200 & 0.51800200 \\ \mathrm{C} & -3.90888600 & -0.99793100 & 0.80002300 \\ \mathrm{C} & -3.66457100 & -1.28804000 & -0.69122300 \\ \mathrm{C} & 2.12373400 & -0.66175800 & 2.41722700 \\ \mathrm{H} & 2.67020000 & -3.03905200 & -1.54700900 \\ \mathrm{H} & 3.39210700 & -2.83383400 & 1.11940400 \\ \mathrm{H} & -4.40402100 & -0.81833000 & -1.34590600 \\ \mathrm{H} & -3.61246500 & -2.35615600 & -0.91931200 \\ \mathrm{H} & -4.81134600 & -0.40804300 & 0.98032600 \\ \mathrm{H} & -3.94670500 & -1.90395500 & 1.41165000 \\ \mathrm{H} & 3.11781700 & -0.85447000 & 2.82862500 \\ \mathrm{H} & 1.92484300 & 0.41030500 & 2.45009900 \\ \mathrm{H} & 1.37326900 & -1.17866500 & 3.02430000 \\ \mathrm{H} & -0.27554500 & -1.08544200 & -2.16395800 \\ \mathrm{H} & 1.06186100 & -0.04052600 & -2.70480200 \\ \mathrm{H} & 1.11561300 & -1.78986100 & -3.04650700 \\ \mathrm{H} & -1.00646300 & 3.12583700 & -0.65507500 \\ \mathrm{H} & -0.82638100 & 3.15728200 & 1.17336600 \\ \mathrm{~N} & 1.59365900 & 2.82333400 & -1.18829900 \\ \mathrm{H} & 1.45901300 & 3.70653700 & -1.66443200 \\ \mathrm{H} & 2.52089400 & 2.43607900 & -1.28566200 \\ \mathrm{H} & 1.59169600 & 2.79772900 & 0.97184400\end{array}$

TS1 $_{\text {NH2 }}$

$\mathrm{E}=-2333.09290769 \mathrm{au} \quad \mathrm{ZPE}=169.7 \mathrm{kcal} / \mathrm{mol}$

$\begin{array}{lrrr}\mathrm{Cu} & -0.10184400 & 0.73461000 & -0.46274000 \\ \mathrm{C} & -1.93858800 & 1.47708600 & -0.53035200 \\ \mathrm{~B} & -1.72111200 & -0.30568500 & 0.14003100 \\ \mathrm{C} & -0.89243800 & 2.45753100 & -0.99766000 \\ \mathrm{C} & 1.35712500 & -1.99085100 & -1.81997400 \\ \mathrm{O} & -2.11846100 & -0.50574500 & 1.45606700 \\ \mathrm{O} & -2.12882800 & -1.36537000 & -0.67740700 \\ \mathrm{C} & 1.47901200 & -0.16985000 & -0.11358700 \\ \mathrm{~N} & 2.01465700 & -1.28601100 & -0.72105300 \\ \mathrm{~N} & 2.41181900 & 0.15734700 & 0.85099700 \\ \mathrm{C} & 3.25122100 & -1.62850900 & -0.15921000 \\ \mathrm{C} & 3.49707300 & -0.72728500 & 0.83062400 \\ \mathrm{C} & -2.77127000 & -1.82389500 & 1.57898200 \\ \mathrm{C} & -2.92544600 & -2.32028100 & 0.12509000 \\ \mathrm{C} & 2.25792000 & 1.28288100 & 1.77209000 \\ \mathrm{H} & 3.83019600 & -2.46669200 & -0.50708900 \\ \mathrm{H} & 4.32664500 & -0.63989100 & 1.51114900 \\ \mathrm{H} & -3.96056900 & -2.28466600 & -0.22717600 \\ \mathrm{H} & -2.52833000 & -3.32589300 & -0.03152900 \\ \mathrm{H} & -3.72707500 & -1.69115800 & 2.09101000\end{array}$




$\begin{array}{rrrr}\mathrm{H} & -2.12872600 & -2.47392500 & 2.18019400 \\ \mathrm{H} & 1.39845900 & 1.87706800 & 1.45293400 \\ \mathrm{H} & 2.08188300 & 0.92922700 & 2.79300500 \\ \mathrm{H} & 3.15378300 & 1.91104400 & 1.75552900 \\ \mathrm{H} & 0.27551800 & -1.95180400 & -1.66804400 \\ \mathrm{H} & 1.60018600 & -1.53088700 & -2.78398900 \\ \mathrm{H} & 1.68271000 & -3.03382600 & -1.82979200 \\ \mathrm{H} & -2.62574300 & 1.17327700 & -1.31970900 \\ \mathrm{H} & -2.46557700 & 1.79249700 & 0.37435000 \\ \mathrm{H} & -1.05778600 & 2.77829500 & -2.03448200 \\ \mathrm{~N} & -0.59902000 & 3.50460700 & -0.02593500 \\ \mathrm{H} & 0.31505100 & 3.92440100 & -0.16066300 \\ \mathrm{H} & -1.31312500 & 4.23403600 & 0.05026500\end{array}$

$2 \mathrm{~A}_{\mathrm{NH} 2}$

$\mathrm{E}=-2333.14450110 \mathrm{au} \quad \mathrm{ZPE}=171.3 \mathrm{kcal} / \mathrm{mol}$

$\begin{array}{lrrr}\mathrm{B} & 2.79216900 & -0.00484900 & -0.24914700 \\ \mathrm{C} & -1.91222700 & -0.21085900 & 0.02075600 \\ \mathrm{C} & -3.46145600 & 1.50021900 & 0.97311700 \\ \mathrm{C} & -3.42148900 & -1.91728800 & -0.25434200 \\ \mathrm{C} & 1.20059700 & 2.00737400 & -0.04078500 \\ \mathrm{C} & 2.36641800 & 1.35759700 & -0.86539500 \\ \mathrm{C} & -4.09969400 & -0.86116700 & 0.27604300 \\ \mathrm{C} & 3.84738100 & -1.54143000 & 1.15481200 \\ \mathrm{C} & 2.86275800 & -2.29393800 & 0.22697100 \\ \mathrm{C} & -1.01811300 & -2.33312100 & -0.95972300 \\ \mathrm{Cu} & -0.35784600 & 0.85634400 & 0.00932000 \\ \mathrm{~N} & -2.09236800 & -1.50673500 & -0.40378100 \\ \mathrm{~N} & -3.16866400 & 0.16960400 & 0.43699000 \\ \mathrm{O} & 3.73828900 & -0.13380900 & 0.74698700 \\ \mathrm{O} & 2.24191700 & -1.23724500 & -0.59282900 \\ \mathrm{H} & -3.78057800 & 1.43635900 & 2.01769800 \\ \mathrm{H} & -2.54144000 & 2.08411300 & 0.91052800 \\ \mathrm{H} & -4.24163600 & 1.98911500 & 0.38250400 \\ \mathrm{H} & -3.75932000 & -2.90064700 & -0.53361400 \\ \mathrm{H} & 1.58244700 & 2.13133400 & 0.98591700 \\ \mathrm{H} & 3.23604000 & 2.03653100 & -0.89931200 \\ \mathrm{H} & 2.04053200 & 1.20675900 & -1.90462000 \\ \mathrm{H} & -5.13662700 & -0.75536700 & 0.54568200 \\ \mathrm{H} & 3.57856500 & -1.61885600 & 2.21150200 \\ \mathrm{H} & 4.88393500 & -1.86196700 & 1.02490900 \\ \mathrm{H} & 3.36197900 & -3.00075000 & -0.44112000 \\ \mathrm{H} & 2.07528100 & -2.81579100 & 0.77741800 \\ \mathrm{H} & -1.23144400 & -2.58604700 & -2.00294400 \\ \mathrm{H} & -0.91223500 & -3.25582700 & -0.38057300 \\ \mathrm{H} & -0.08571900 & -1.76720300 & -0.91126000 \\ \mathrm{~N} & 0.86563100 & 3.36934000 & -0.50219200 \\ \mathrm{H} & 0.40757800 & 3.37644800 & -1.41095200 \\ \mathrm{H} & 1.68043500 & 3.99072300 & -0.53541900\end{array}$

\section{IN1 NH2 $^{\prime}$}

$\mathrm{E}=-2333.13094796 \mathrm{au} \quad \mathrm{ZPE}=170.4 \mathrm{kcal} / \mathrm{mol}$

$\begin{array}{lrrr}\mathrm{Cu} & -0.07623900 & 0.65609500 & -0.33258700 \\ \mathrm{C} & -1.27712000 & 2.25656400 & -0.92836100 \\ \mathrm{~B} & -1.64870300 & -0.53632400 & 0.14617400 \\ \mathrm{C} & 0.06817900 & 2.58919400 & -1.08085500 \\ \mathrm{C} & 1.28986200 & -2.22161200 & -1.39534100 \\ \mathrm{O} & -2.81138100 & -0.08688100 & 0.79216900 \\ \mathrm{O} & -1.78441900 & -1.89920800 & -0.14501500 \\ \mathrm{C} & 1.68005100 & -0.14156300 & -0.04492000 \\ \mathrm{~N} & 2.09451600 & -1.37117600 & -0.51173100 \\ \mathrm{~N} & 2.73644400 & 0.26432400 & 0.74559900 \\ \mathrm{C} & 3.36237200 & -1.71078000 & -0.02795800 \\ \mathrm{C} & 3.76596600 & -0.68501500 & 0.76961800 \\ \mathrm{C} & -3.76900100 & -1.20396100 & 0.99309500 \\ \mathrm{C} & -3.11424500 & -2.40364100 & 0.28833300 \\ \mathrm{C} & 2.75986900 & 1.51793100 & 1.49341400 \\ \mathrm{H} & 0.52420400 & 3.29393500 & -0.38434800 \\ \mathrm{H} & 3.85245500 & -2.63242300 & -0.29103500 \\ \mathrm{H} & 4.66832300 & -0.54879300 & 1.34072500 \\ \mathrm{H} & -3.66302300 & -2.72988800 & -0.59968900 \\ \mathrm{H} & -2.95921000 & -3.26019100 & 0.94906300 \\ \mathrm{H} & -4.72905700 & -0.91849700 & 0.55579700 \\ \mathrm{H} & -3.89715800 & -1.35821600 & 2.06824800\end{array}$
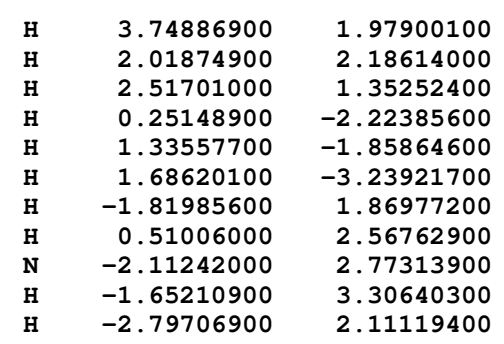

1.42035700

1.05526600

2. 54849200

$-1.05396100$

$-2.42690100$

$-1.36051200$

$-1.78374400$

$-2.07397500$

0.07239500

0.80027800

0.42686000

TS1 NH2' $^{\prime}$

$\mathrm{E}=-2333.09621672 \mathrm{au} \quad \mathrm{ZPE}=169.7 \mathrm{kcal} / \mathrm{mol}$

$\begin{array}{lrrr}\mathrm{C} & -1.58771200 & 0.06123800 & 0.11085900 \\ \mathrm{C} & -2.61932900 & 0.97162500 & -1.97299500 \\ \mathrm{C} & -3.32131800 & -1.28489500 & 0.78322500 \\ \mathrm{C} & 1.01637600 & 2.68487300 & 0.01004400 \\ \mathrm{C} & 1.96151800 & 1.53420300 & 0.19847100 \\ \mathrm{C} & -3.70108900 & -0.69116800 & -0.38178300 \\ \mathrm{C} & -1.23430900 & -1.21510000 & 2.23368900 \\ \mathrm{Cu} & 0.03154800 & 0.97745000 & 0.04363500 \\ \mathrm{~N} & -2.02957200 & -0.82864000 & 1.06806100 \\ \mathrm{~N} & -2.63866400 & 0.12760100 & -0.78086200 \\ \mathrm{H} & -3.61986500 & 1.36918100 & -2.16214500 \\ \mathrm{H} & -1.92702400 & 1.79684100 & -1.78440200 \\ \mathrm{H} & -2.27787300 & 0.41325300 & -2.85029500 \\ \mathrm{H} & -3.84313300 & -1.97565300 & 1.42297400 \\ \mathrm{H} & 2.72333500 & 1.49022700 & -0.59495400 \\ \mathrm{H} & -4.61000600 & -0.77804500 & -0.95177700 \\ \mathrm{H} & -1.60341000 & -2.16752600 & 2.62130400 \\ \mathrm{H} & -1.30057400 & -0.45705500 & 3.02075500 \\ \mathrm{H} & -0.18877700 & -1.32396200 & 1.93411100 \\ \mathrm{~B} & 1.57027200 & -0.30072300 & -0.27087300 \\ \mathrm{O} & 2.09894900 & -1.25408200 & 0.60439600 \\ \mathrm{O} & 1.76263600 & -0.68142500 & -1.59746300 \\ \mathrm{C} & 2.73789400 & -2.33934300 & -0.17715400 \\ \mathrm{C} & 2.38391200 & -2.01876900 & -1.64542200 \\ \mathrm{H} & 3.81423000 & -2.31356500 & 0.01586600 \\ \mathrm{H} & 2.33501500 & -3.29562400 & 0.16398800 \\ \mathrm{H} & 3.25981600 & -1.97206900 & -2.29689500 \\ \mathrm{H} & 1.66035800 & -2.72242700 & -2.06727600 \\ \mathrm{H} & 1.14483200 & 3.23361000 & -0.92356100 \\ \mathrm{H} & 0.93664100 & 3.33601600 & 0.88079800 \\ \mathrm{~N} & 2.42595800 & 1.41141100 & 1.55602600 \\ \mathrm{H} & 2.91232000 & 0.55812100 & 1.79626700 \\ \mathrm{H} & 2.80547100 & 2.26171700 & 1.95255000\end{array}$

$2 \mathrm{~A}_{\mathrm{NH} 2^{\prime}}$

$\mathrm{E}=-2333.14342471 \mathrm{au} \quad \mathrm{ZPE}=171.0 \mathrm{kcal} / \mathrm{mol}$

$\begin{array}{lrrr}\mathrm{B} & -2.55570800 & 0.22286500 & -0.07379100 \\ \mathrm{C} & 1.98456500 & -0.07920100 & -0.05534700 \\ \mathrm{C} & 3.31762100 & -1.71764100 & 1.27873000 \\ \mathrm{C} & 3.64450600 & 1.45663400 & -0.44941300 \\ \mathrm{C} & -1.33371400 & -1.96116200 & -0.11750800 \\ \mathrm{C} & -2.43797100 & -1.14375400 & -0.82799900 \\ \mathrm{C} & 4.19287200 & 0.45284500 & 0.29068000 \\ \mathrm{C} & -3.09415800 & 1.83241600 & 1.51976000 \\ \mathrm{C} & -2.13667600 & 2.45711100 & 0.47574300 \\ \mathrm{C} & 1.34784900 & 1.91887500 & -1.42584100 \\ \mathrm{Cu} & 0.34140500 & -1.00572700 & -0.06152400 \\ \mathrm{~N} & 2.30173600 & 1.12028500 & -0.65070000 \\ \mathrm{~N} & 3.17171500 & -0.47419800 & 0.52167900 \\ \mathrm{O} & -3.29315600 & 0.45003800 & 1.06457500 \\ \mathrm{O} & -1.87951500 & 1.37212200 & -0.48651800 \\ \mathrm{H} & 3.56651500 & -1.50989500 & 2.32379300 \\ \mathrm{H} & 2.35965700 & -2.23782600 & 1.23189500 \\ \mathrm{H} & 4.09473400 & -2.34840700 & 0.83724300 \\ \mathrm{H} & 4.08048900 & 2.35942400 & -0.84180800 \\ \mathrm{H} & -1.71673300 & -2.24139800 & 0.87594300 \\ \mathrm{H} & -2.06816700 & -0.92662100 & -1.84923800 \\ \mathrm{H} & 5.19350600 & 0.32098000 & 0.66543700 \\ \mathrm{H} & -2.66722600 & 1.80853100 & 2.52568600 \\ \mathrm{H} & -4.06686600 & 2.32945900 & 1.55612200\end{array}$




$\begin{array}{lrrr}\mathrm{H} & -2.58035300 & 3.29915200 & -0.06204200 \\ \mathrm{H} & -1.18363800 & 2.77177700 & 0.91088200 \\ \mathrm{H} & 1.58684100 & 1.87319300 & -2.49305300 \\ \mathrm{H} & 1.37828500 & 2.96133400 & -1.09522900 \\ \mathrm{H} & 0.34496000 & 1.52033500 & -1.26161900 \\ \mathrm{H} & -1.21384500 & -2.90972100 & -0.67384100 \\ \mathrm{~N} & -3.71556700 & -1.87796700 & -0.83832500 \\ \mathrm{H} & -4.46568800 & -1.42371000 & -1.35413600 \\ \mathrm{H} & -3.59786000 & -2.84571500 & -1.12875700\end{array}$

\section{$\mathrm{CH}_{2} \mathrm{CHNMe}_{2}$}

$\mathrm{E}=-212.545804800 \mathrm{au} \quad \mathrm{ZPE}=79.0 \mathrm{kcal} / \mathrm{mol}$

$\begin{array}{lrrr}\mathrm{C} & 2.02655800 & -0.12581400 & 0.08302900 \\ \mathrm{C} & 0.80915800 & -0.67093200 & -0.08120000 \\ \mathrm{~N} & -0.40867600 & -0.02232300 & -0.15298600 \\ \mathrm{C} & -1.64348700 & -0.76611300 & 0.09098000 \\ \mathrm{H} & -2.46001200 & -0.34359200 & -0.50521100 \\ \mathrm{H} & -1.50674700 & -1.80873900 & -0.20957100 \\ \mathrm{H} & -1.94983800 & -0.75317000 & 1.14979100 \\ \mathrm{C} & -0.45974200 & 1.42856100 & -0.00971000 \\ \mathrm{H} & -0.26532200 & 1.76104100 & 1.02330000 \\ \mathrm{H} & 0.28718600 & 1.89201200 & -0.66248700 \\ \mathrm{H} & -1.44887400 & 1.78713400 & -0.30827100 \\ \mathrm{H} & 2.18598900 & 0.93738900 & 0.23269200 \\ \mathrm{H} & 2.90213500 & -0.75898500 & 0.05219500 \\ \mathrm{H} & 0.72129300 & -1.75104000 & -0.20013400\end{array}$

\section{IN1 $1_{\mathrm{NMe} 2}$}

$\mathrm{E}=-2411.71631259 \mathrm{au} \quad \mathrm{ZPE}=205.4 \mathrm{kcal} / \mathrm{mol}$

$\begin{array}{lrrr}\mathrm{Cu} & 0.03233100 & -0.55031400 & -0.32627300 \\ \mathrm{C} & 0.63758400 & -2.40169800 & -0.68501500 \\ \mathrm{~B} & -1.96781000 & -0.74797500 & -0.13310200 \\ \mathrm{C} & 1.77876600 & -1.58871400 & -0.64034100 \\ \mathrm{C} & -0.24423500 & 1.42095300 & 2.35482100 \\ \mathrm{O} & -2.88384800 & -0.99069200 & -1.15002200 \\ \mathrm{O} & -2.63381900 & -0.60407900 & 1.09156800 \\ \mathrm{C} & 0.39859700 & 1.32772400 & -0.06198800 \\ \mathrm{~N} & 0.17352900 & 2.04292700 & 1.09339000 \\ \mathrm{~N} & 0.71698100 & 2.30221900 & -0.98408200 \\ \mathrm{C} & 0.34157900 & 3.41707900 & 0.89067100 \\ \mathrm{C} & 0.67769000 & 3.58328600 & -0.41764700 \\ \mathrm{C} & -4.26554800 & -1.01619200 & -0.60808100 \\ \mathrm{C} & -4.09535300 & -0.80559600 & 0.90684400 \\ \mathrm{C} & 0.98837900 & 2.02342700 & -2.39203300 \\ \mathrm{H} & 0.21371400 & 4.13616800 & 1.68134100 \\ \mathrm{H} & 0.88927800 & 4.47387600 & -0.98397400 \\ \mathrm{H} & -4.39866400 & -1.67440700 & 1.49739600 \\ \mathrm{H} & -4.61546700 & 0.08057600 & 1.28007200 \\ \mathrm{H} & -4.71458800 & -1.98028700 & -0.85981600 \\ \mathrm{H} & -4.83829800 & -0.21847900 & -1.08940000 \\ \mathrm{H} & 1.70408700 & 2.75150100 & -2.78187700 \\ \mathrm{H} & 1.41583700 & 1.02307000 & -2.47257100 \\ \mathrm{H} & 0.06891700 & 2.06546100 & -2.98506900 \\ \mathrm{H} & -1.10510700 & 0.77082700 & 2.17183400 \\ \mathrm{H} & 0.57285200 & 0.83082100 & 2.77883900 \\ \mathrm{H} & -0.52030000 & 2.20711700 & 3.06088400 \\ \mathrm{H} & 0.39463200 & -3.03496800 & 0.16144500 \\ \mathrm{H} & 0.25393600 & -2.74176300 & -1.64435400 \\ \mathrm{~N} & 2.65354100 & -1.60481800 & 0.49558400 \\ \mathrm{H} & 2.23620100 & -1.28874100 & -1.59584600 \\ \mathrm{C} & 3.37118200 & -2.87595800 & 0.71107700 \\ \mathrm{H} & 4.14833800 & -3.06418000 & -0.05484100 \\ \mathrm{H} & 3.85581200 & -2.85935900 & 1.69372100 \\ \mathrm{H} & 2.66181400 & -3.70495500 & 0.68913200 \\ \mathrm{C} & 3.53230700 & -0.43709800 & 0.59612700 \\ \mathrm{H} & 4.08330100 & -0.46995500 & 1.54243300 \\ \mathrm{H} & 4.27474600 & -0.38354800 & -0.22603400 \\ \mathrm{H} & 2.92772700 & 0.47279300 & 0.57089400\end{array}$

\section{TS1 NMe2}

$\mathrm{E}=-2411.68801344 \mathrm{au} \quad \mathrm{ZPE}=204.9 \mathrm{kcal} / \mathrm{mol}$

$\begin{array}{llll}\mathrm{Cu} & 0.30620400 & -0.14063600 & -0.32902000 \\ \mathrm{C} & 1.81541400 & 1.14225100 & -0.40606600\end{array}$

$\begin{array}{lr}\text { B } & 0.08524000 \\ \mathrm{C} & 2.20621000 \\ \mathrm{C} & -2.53934300 \\ \mathrm{O} & 0.03977900 \\ \mathrm{O} & -0.61690300 \\ \mathrm{C} & -1.28809900 \\ \mathrm{~N} & -2.42407900 \\ \mathrm{~N} & -1.61339900 \\ \mathrm{C} & -3.41579800 \\ \mathrm{C} & -2.91049100 \\ \mathrm{C} & -0.81857000 \\ \mathrm{C} & -1.11833100 \\ \mathrm{C} & -0.71762000 \\ \mathrm{H} & -4.37082900 \\ \mathrm{H} & -3.34708000 \\ \mathrm{H} & -0.58298800 \\ \mathrm{H} & -2.18425700 \\ \mathrm{H} & -0.27286400 \\ \mathrm{H} & -1.72031900 \\ \mathrm{H} & 0.30628900 \\ \mathrm{H} & -0.95534800 \\ \mathrm{H} & -0.79635600 \\ \mathrm{H} & -2.06640300 \\ \mathrm{H} & -2.05072400 \\ \mathrm{H} & -3.59550600 \\ \mathrm{H} & 1.88978000 \\ \mathrm{H} & 2.29762200 \\ \mathrm{H} & 2.48828000 \\ \mathrm{~N} & 3.12637600 \\ \mathrm{C} & 3.08068000 \\ \mathrm{H} & 3.45323100 \\ \mathrm{H} & 3.69277000 \\ \mathrm{H} & 2.04367700 \\ \mathrm{C} & 4.51116400 \\ \mathrm{H} & 5.12705100 \\ \mathrm{H} & 4.98399800 \\ \mathrm{H} & 4.51727300\end{array}$

1.80424300

$-0.23972800$

$-0.20620100$

2. 31544000

2. 61916000

$-1.06348900$

$-1.02728500$

$-1.95177800$

$-1.87995600$

$-2.45454400$

3. 51738000

3. 81105300

$-2.27913600$

$-1.99836900$

$-3.15839600$

4.68847900

3. 92364900

4. 32310300

3.28673400

$-2.05672800$

$-1.68592700$

$-3.34249400$

0.76279100

$-0.69145100$

$-0.05424500$

1. 90180800

1.45780800

$-0.28023300$

$-0.93556400$

$-2.39470000$

$-2.74697100$

$-2.86938800$

$-2.72678300$

$-0.43124400$

$-0.92142600$

$-0.60456900$

0.64532100
0.14850400

$-0.85215200$

$-2.06741400$

1. 43879000

$-0.74454600$

$-0.08022400$

$-0.86273000$

0.92504900

$-0.36280600$

0.76240900

1. 46220400

$-0.02354000$

2. 03167700

$-0.84515700$

1. 44993000

$-0.39790100$

$-0.23387700$

1. 95828900

2. 03690200

1. 72466400

2. 92068000

2. 27316400

$-1.88747900$

$-2.91914700$

$-2.30087800$

$-1.18432500$

0.52186900

$-1.91823600$

0.03288500

$-0.11061700$

$-1.09460100$

0.66752200

$-0.01213600$

$-0.03005400$

0.73421500

$-1.01842700$

0.16312500

$2 \mathbf{A}_{\mathrm{NMe} 2}$

$\mathrm{E}=-2411.73790988 \mathrm{au} \quad \mathrm{ZPE}=206.6 \mathrm{kcal} / \mathrm{mol}$

$\begin{array}{lrrr}\text { B } & -2.46516400 & -1.03020500 & 0.20421600 \\ \mathrm{C} & 2.09746300 & -0.13668000 & -0.06852000 \\ \mathrm{C} & 3.21599300 & 1.76282200 & -1.24443400 \\ \mathrm{C} & 3.96374500 & -1.39000700 & 0.39242700 \\ \mathrm{C} & -1.45713700 & 1.28423100 & -0.22764200 \\ \mathrm{C} & -2.40905600 & 0.43636700 & 0.70555000 \\ \mathrm{C} & 4.38091200 & -0.27701500 & -0.27348000 \\ \mathrm{C} & -3.07007100 & -2.88539500 & -1.07651400 \\ \mathrm{C} & -1.96263900 & -3.29814700 & -0.07682700 \\ \mathrm{C} & 1.72157600 & -2.27361300 & 1.18418200 \\ \mathrm{Cu} & 0.33653700 & 0.53881900 & -0.15888800 \\ \mathrm{~N} & 2.57320200 & -1.29086800 & 0.50927200 \\ \mathrm{~N} & 3.23503700 & 0.47597700 & -0.54660700 \\ \mathrm{O} & -3.32715000 & -1.46958000 & -0.78081000 \\ \mathrm{O} & -1.63405700 & -2.05708500 & 0.64799000 \\ \mathrm{H} & 3.55211400 & 1.64881200 & -2.27936700 \\ \mathrm{H} & 2.18499900 & 2.12052400 & -1.23595000 \\ \mathrm{H} & 3.85494400 & 2.48720400 & -0.73109100 \\ \mathrm{H} & 4.52005400 & -2.22341000 & 0.78620100 \\ \mathrm{H} & -1.85893300 & 1.17100200 & -1.24842000 \\ \mathrm{H} & -3.43321000 & 0.84576400 & 0.71921100 \\ \mathrm{H} & -2.03745200 & 0.45774600 & 1.73578900 \\ \mathrm{H} & 5.36718100 & 0.03729600 & -0.56940600 \\ \mathrm{H} & -2.75329300 & -2.97065900 & -2.11932300 \\ \mathrm{H} & -3.99845400 & -3.44472100 & -0.93788700 \\ \mathrm{H} & -2.30043000 & -4.04242900 & 0.64926800 \\ \mathrm{H} & -1.05828500 & -3.66350400 & -0.57081800 \\ \mathrm{H} & 1.96269400 & -2.31893500 & 2.25080700 \\ \mathrm{H} & 1.86524500 & -3.26407200 & 0.74112900 \\ \mathrm{H} & 0.67908700 & -1.97283500 & 1.06450000 \\ \mathrm{~N} & -1.50576600 & 2.73584900 & 0.03775200 \\ \mathrm{C} & -2.80363300 & 3.38626600 & -0.17651100 \\ \mathrm{H} & -3.56381400 & 3.15804600 & 0.59706300 \\ \mathrm{H} & -2.67347000 & 4.47665300 & -0.19517500 \\ \mathrm{H} & -3.20714500 & 3.07189300 & -1.14480100\end{array}$




$\begin{array}{lrll}\mathrm{C} & -0.84406500 & 3.18720300 & 1.25941900 \\ \mathrm{H} & 0.16975600 & 2.77214100 & 1.28654400 \\ \mathrm{H} & -0.77555500 & 4.28384400 & 1.26521300 \\ \mathrm{H} & -1.35595800 & 2.88531900 & 2.19692100\end{array}$

\section{IN1 NMe2 $^{\prime}$}

$\mathrm{E}=-2411.72313832 \mathrm{au} \quad \mathrm{ZPE}=205.8 \mathrm{kcal} / \mathrm{mol}$

$\begin{array}{rrrr}\mathrm{Cu} & 0.08907500 & -0.44069900 & -0.41193600 \\ \mathrm{C} & 1.71315800 & -1.55740200 & -1.19440000 \\ \mathrm{~B} & 0.89661100 & 1.32659800 & 0.13548700 \\ \mathrm{C} & 0.51084700 & -2.27411500 & -1.22359900 \\ \mathrm{C} & -2.36816200 & 1.48841800 & -1.68941500 \\ \mathrm{O} & 1.68736300 & 1.60345200 & 1.25518300 \\ \mathrm{O} & 0.60110900 & 2.52616300 & -0.53352700 \\ \mathrm{C} & -1.83723300 & -0.34637000 & -0.06604900 \\ \mathrm{~N} & -2.72854400 & 0.54130100 & -0.62962400 \\ \mathrm{~N} & -2.60436500 & -1.00829800 & 0.87022400 \\ \mathrm{C} & -4.00299900 & 0.43079000 & -0.06087800 \\ \mathrm{C} & -3.92527800 & -0.54025000 & 0.88887500 \\ \mathrm{C} & 1.92005000 & 3.06469100 & 1.38551200 \\ \mathrm{C} & 1.24793100 & 3.67868500 & 0.14647300 \\ \mathrm{C} & -2.08059400 & -2.03531100 & 1.76608400 \\ \mathrm{H} & 0.33788200 & -3.09983100 & -0.53731600 \\ \mathrm{H} & -4.83217200 & 1.03915200 & -0.37917100 \\ \mathrm{H} & -4.67015900 & -0.93014700 & 1.56131700 \\ \mathrm{H} & 1.96123000 & 4.13138800 & -0.54815600 \\ \mathrm{H} & 0.47550900 & 4.41176600 & 0.39380600 \\ \mathrm{H} & 2.99842400 & 3.23980000 & 1.42089400 \\ \mathrm{H} & 1.47317900 & 3.40137200 & 2.32532900 \\ \mathrm{H} & -2.82733700 & -2.82103500 & 1.90955800 \\ \mathrm{H} & -1.18982200 & -2.46626100 & 1.30849400 \\ \mathrm{H} & -1.81191400 & -1.61043100 & 2.73902000 \\ \mathrm{H} & -1.39895600 & 1.93933100 & -1.45916800 \\ \mathrm{H} & -2.31750900 & 0.98267100 & -2.65888300 \\ \mathrm{H} & -3.12923600 & 2.27092600 & -1.73858400 \\ \mathrm{H} & 1.99593100 & -1.00605900 & -2.08471700 \\ \mathrm{H} & -0.00520900 & -2.35806900 & -2.17736600 \\ \mathrm{~N} & 2.80449600 & -1.80273200 & -0.35793600 \\ \mathrm{C} & 2.58957500 & -2.57509800 & 0.86166400 \\ \mathrm{H} & 2.11908600 & -3.53393800 & 0.62516500 \\ \mathrm{H} & 3.55608900 & -2.77692800 & 1.33194900 \\ \mathrm{H} & 1.95513500 & -2.03986000 & 1.59192000 \\ \mathrm{C} & 3.80462700 & -0.73222700 & -0.24890300 \\ \mathrm{H} & 4.74131700 & -1.15055700 & 0.13396700 \\ \mathrm{H} & 3.99598300 & -0.31657900 & -1.24302800 \\ \mathrm{H} & 3.46588300 & 0.08089500 & 0.40960000\end{array}$

\section{TS1 $_{\text {NMe2' }}$}

\begin{tabular}{|c|c|c|c|}
\hline \\
\hline \multicolumn{4}{|c|}{ 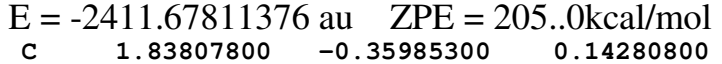 } \\
\hline \multicolumn{4}{|c|}{$\begin{array}{llll}\text { C } & 3.05683500 & -0.61627100 & -2.02291300\end{array}$} \\
\hline \multicolumn{4}{|c|}{$\begin{array}{lrrr}\mathrm{C} & 3.05683500 & -0.61627100 & -2.02291300 \\
\mathrm{C} & 3.63138000 & 0.17560500 & 1.47290100\end{array}$} \\
\hline \multicolumn{4}{|c|}{-1.17631300} \\
\hline \multirow{2}{*}{$\begin{array}{l}\text { C } \\
\text { C }\end{array}$} & -1.93673500 & -0.79499700 & -0.70862900 \\
\hline & 4.10656200 & 0.00067200 & 0.2089190 \\
\hline \multirow{2}{*}{$\begin{array}{l}\mathrm{C} \\
\mathrm{Cu}\end{array}$} & 1.33555000 & 0.05923600 & 2.5589510 \\
\hline & 0.12281400 & -0.75538300 & -0.4738200 \\
\hline \multirow[t]{2}{*}{$\mathbf{N}$} & 2.24909800 & -0.03425600 & 1.4193340 \\
\hline & 3.00674200 & -0.32625300 & -0.5922070 \\
\hline $\mathrm{H}$ & 3.98495500 & -1.14155400 & -2.2632980 \\
\hline & 2.20174600 & -1.25442400 & -2.2624180 \\
\hline & 2.99347000 & 0.30102200 & -2.6168130 \\
\hline $\mathrm{H}$ & 4.14146000 & 0.42505100 & 2.3873230 \\
\hline \multirow[t]{2}{*}{$\mathrm{H}$} & -2.54325700 & -0.21892000 & -1.4170650 \\
\hline & 5.10526300 & 0.08096800 & -0.1846570 \\
\hline $\mathrm{H}$ & 1.80911500 & 0.64500700 & 3.3503120 \\
\hline \multirow{2}{*}{$\begin{array}{l}\mathrm{H} \\
\mathrm{H}\end{array}$} & 1.09473400 & -0.93570500 & 2.9476490 \\
\hline & 0.41410400 & 0.55440600 & 2.2419610 \\
\hline & -1.00658500 & 0.92560400 & -0.3637370 \\
\hline \multirow{2}{*}{$\begin{array}{l}0 \\
0\end{array}$} & -1.24744300 & 1.65223500 & 0.8024120 \\
\hline & -0.95036500 & 1.76852800 & -1.4724460 \\
\hline C & -1.40999800 & 3.08770600 & 0.4641290 \\
\hline \multirow[t]{2}{*}{ C } & -1.04225400 & 3.17497700 & -1.0316360 \\
\hline & -2.44752300 & 3.37206200 & 0.6637000 \\
\hline
\end{tabular}

$\begin{array}{ll}\mathrm{H} & -0.74761000 \\ \mathrm{H} & -1.80155300 \\ \mathrm{H} & -0.07356000 \\ \mathrm{H} & -1.17819900 \\ \mathrm{H} & -1.44141300 \\ \mathrm{~N} & -2.66490500 \\ \mathrm{C} & -3.81339400 \\ \mathrm{H} & -3.50653900 \\ \mathrm{H} & -4.65174400 \\ \mathrm{H} & -4.18573300 \\ \mathrm{C} & -2.84952100 \\ \mathrm{H} & -3.26541800 \\ \mathrm{H} & -3.53438000 \\ \mathrm{H} & -1.89182900\end{array}$

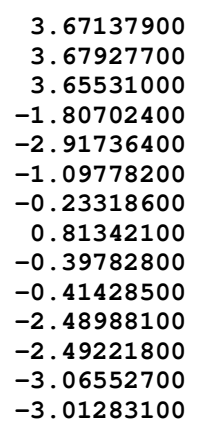

1.10699200 $-1.63371800$ $-1.19943300$ $-2.46714100$ $-1.08216000$

0.50110000

0.76699900

0.71021600

0.06198500

1.78135900

0.91889100

1.93248400

0.26907000

0.95123500

$\mathbf{2 A}_{\mathrm{NMe}}$

$\mathrm{E}=-2411.73748584 \mathrm{au} \quad \mathrm{ZPE}=206.5 \mathrm{kcal} / \mathrm{mol}$

\begin{tabular}{lrrr} 
B & -1.91964200 & 0.78311100 & \multicolumn{1}{c}{0.19279400} \\
$\mathrm{C}$ & 2.36087300 & -0.27958800 & -0.11302300 \\
$\mathrm{C}$ & 3.50886100 & -1.85805300 & 1.44764400 \\
$\mathrm{C}$ & 4.21473600 & 0.83839700 & -0.87582400 \\
$\mathrm{C}$ & -1.20858100 & -1.57173100 & 0.30363100 \\
$\mathrm{C}$ & -2.22616800 & -0.63758300 & -0.39294100 \\
$\mathrm{C}$ & 4.64704100 & -0.09352400 & 0.01893000 \\
$\mathrm{C}$ & -1.78873600 & 2.57381200 & 1.67606200 \\
$\mathrm{C}$ & -0.96628800 & 2.90714800 & 0.40742000 \\
$\mathrm{C}$ & 1.95673600 & 1.50946800 & -1.82032700 \\
$\mathrm{Cu}$ & 0.59298200 & -0.89640900 & 0.12501300 \\
$\mathrm{~N}$ & 2.82325600 & 0.71446000 & -0.94474500 \\
$\mathrm{~N}$ & 3.50857300 & -0.76645300 & 0.47337600 \\
$\mathrm{O}$ & -2.34227400 & 1.23725800 & 1.42178900 \\
$\mathrm{O}$ & -1.13882100 & 1.73273600 & -0.46632900 \\
$\mathrm{H}$ & 3.86481700 & -1.51011200 & 2.42204700 \\
$\mathrm{H}$ & 2.47950000 & -2.20719300 & 1.54211400 \\
$\mathrm{H}$ & 4.14015500 & -2.68166400 & 1.10170000 \\
$\mathrm{H}$ & 4.76105500 & 1.56154600 & -1.45690300 \\
$\mathrm{H}$ & -1.52511400 & -1.69422400 & 1.35073200 \\
$\mathrm{H}$ & -1.97497300 & -0.61710700 & -1.47632000 \\
$\mathrm{H}$ & 5.63876500 & -0.32973200 & 0.36499800 \\
$\mathrm{H}$ & -1.17518400 & 2.52978700 & 2.57954400 \\
$\mathrm{H}$ & -2.61719100 & 3.26766400 & 1.83985200 \\
$\mathrm{H}$ & -1.33448100 & 3.78892100 & -0.12308500 \\
$\mathrm{H}$ & 0.10013100 & 3.03263000 & 0.61650700 \\
$\mathrm{H}$ & 2.08521500 & 1.20786300 & -2.86461600 \\
$\mathrm{H}$ & 2.20010800 & 2.57130600 & -1.72075400 \\
$\mathrm{H}$ & 0.91919100 & 1.34973100 & -1.52145800 \\
$\mathrm{H}$ & -1.25223200 & -2.57162700 & -0.15700200 \\
$\mathrm{~N}$ & -3.64622600 & -1.02043700 & -0.21927800 \\
$\mathrm{C}$ & -4.57633000 & -0.04604800 & -0.79897900 \\
$\mathrm{H}$ & -5.60841300 & -0.33873600 & -0.57402800 \\
$\mathrm{H}$ & -4.40594100 & 0.94028100 & -0.35532300 \\
$\mathrm{H}$ & -4.48356400 & 0.04839000 & -1.90211300 \\
$\mathrm{C}$ & -3.95809200 & -2.38658000 & -0.65072500 \\
$\mathrm{H}$ & -3.80786200 & -2.54462600 & -1.74047700 \\
$\mathrm{H}$ & -3.33065700 & -3.10078800 & -0.11480400 \\
$\mathrm{H}$ & -5.00636200 & -2.60924800 & -0.42032200
\end{tabular}

3B

$\mathrm{E}=-2260.62719070 \mathrm{au} \quad \mathrm{ZPE}=155.2 \mathrm{kcal} / \mathrm{mol}$

$\begin{array}{crrr}\text { B } & 0.70764800 & 0.93350100 & 0.25234700 \\ \text { C } & 0.41386600 & -0.55942500 & 0.35260100 \\ \text { C } & -2.87136200 & -1.66746400 & 0.36038300 \\ \text { C } & -0.59675800 & -1.14704900 & -0.65751900 \\ \text { C } & -4.19295000 & -1.32641200 & 0.67114800 \\ \text { C } & -4.71269200 & -0.09191000 & 0.26566600 \\ \text { C } & -3.89878500 & 0.79588500 & -0.44991100 \\ \text { C } & -2.57779500 & 0.45295000 & -0.75577600 \\ \text { C } & -2.04280200 & -0.78647200 & -0.35579400 \\ \text { C } & 1.34107300 & 3.10859300 & 0.83505600 \\ \text { C } & 1.07188400 & 3.04612200 & -0.68780600 \\ \text { Cu } & 2.21201000 & -1.16029000 & 0.04923900 \\ \text { O } & 1.19847600 & 1.72451500 & 1.29525100 \\ \text { O } & 0.56930200 & 1.68894800 & -0.91938200 \\ \text { H } & 0.14780600 & -0.84588500 & 1.37965300\end{array}$




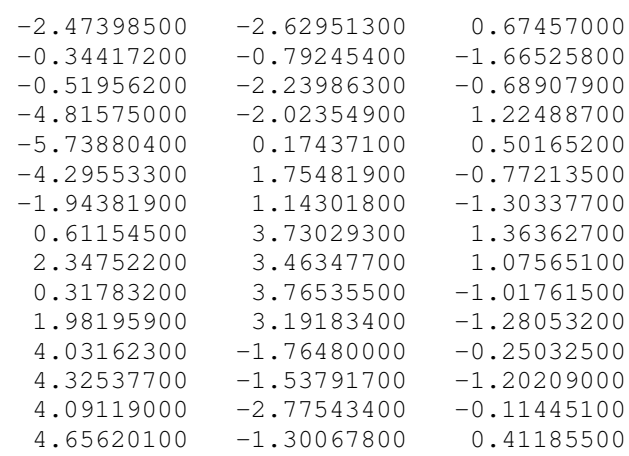

0.67457000

$-1.66525800$

$-0.68907900$

1.22488700

0.50165200

$-0.77213500$

$-1.30337700$

1.36362700

1.07565100

$-1.01761500$

$-1.28053200$

$-0.25032500$

$-1.20209000$

$-0.11445100$

0.41185500

3C

\begin{tabular}{|c|c|c|c|}
\hline $\mathrm{E}$ & 564.2039846 & au $\quad \mathrm{ZPE}=$ & $7.5 \mathrm{kcal} / \mathrm{mol}$ \\
\hline B & -1.73087100 & 0.63332000 & 0.12785400 \\
\hline $\mathrm{C}$ & -0.74855400 & 1.85069600 & 0.06433800 \\
\hline $\mathrm{C}$ & 2.48654900 & 0.96854800 & 0.43295600 \\
\hline C & 0.44784100 & 1.68126200 & -0.9025750 \\
\hline $\mathrm{C}$ & 3.39216000 & 0.00146600 & 0.8816810 \\
\hline $\mathrm{C}$ & 3.27014700 & -1.32635900 & 0.4545330 \\
\hline $\mathrm{C}$ & 2.23738500 & -1.67690600 & -0.4228380 \\
\hline $\mathrm{C}$ & 1.33181900 & -0.70745200 & -0.8691600 \\
\hline $\mathrm{C}$ & 1.44348900 & 0.62975000 & -0.44 \\
\hline $\mathrm{C}$ & -3.30390400 & -0.87384700 & 0.933833 \\
\hline $\mathrm{C}$ & -2.99932500 & -1.19254900 & -0.551693 \\
\hline O & -2.44895200 & 0.28733700 & 1.2 \\
\hline $\mathrm{O}$ & -2.00215400 & -0.18041500 & -0.95 \\
\hline $\mathrm{H}$ & -0.39193400 & 2.08682100 & 1.07 \\
\hline $\mathrm{H}$ & 2.59097200 & 1.99926700 & 0.7 \\
\hline $\mathrm{H}$ & 0.06752000 & 1.42097400 & -1.8 \\
\hline $\mathrm{H}$ & 0.96483000 & 2.64489000 & -1.00 \\
\hline $\mathrm{H}$ & 4.19312700 & 0.28391000 & 1.5 \\
\hline $\mathrm{H}$ & 3.97451700 & -2.07757400 & 0.7 \\
\hline $\mathrm{H}$ & 2.13989100 & -2.70342000 & -0.76 \\
\hline $\mathrm{H}$ & 0.52925400 & -0.98051200 & -1.54 \\
\hline $\mathrm{H}$ & -3.035349 & -1.68855000 & 1.6 \\
\hline $\mathrm{H}$ & -4.34479600 & -0.59067900 & 1.1 \\
\hline $\mathrm{H}$ & -2.56005000 & -2.18234500 & $-0.69464^{\circ}$ \\
\hline $\mathrm{H}$ & -3.87259000 & -1.08852800 & -1.199 \\
\hline $\mathrm{H}$ & -1.33783100 & 2.72611600 & -0 . \\
\hline
\end{tabular}

$\begin{array}{lrrr}\mathrm{H} & -2.42995800 & 0.70528500 & -1.81126300 \\ \mathrm{H} & -1.74598900 & -0.24470600 & 2.24549300\end{array}$

$3 \mathbf{E}$

$\mathrm{E}=-524.459198531 \mathrm{au} \quad \mathrm{ZPE}=153.1 \mathrm{kcal} / \mathrm{mol}$

C $\quad-0.68909100 \quad 1.86802300 \quad 0.36372100$

$\begin{array}{llll}\text { C } & 2.58668300 & 0.88516000 & 0.44350500\end{array}$

C $\quad 0.49110900 \quad 1.81840700 \quad-0.64280300$

C $\quad 3.47456300 \quad-0.16070900 \quad 0.71831000$

$3.25402600 \quad-1.43059700 \quad 0.17200500$

$2.14001500-1.64477500-0.64841000$

$1.25403000-0.59663000-0.92094600$

$1.46426100 \quad 0.68426900 \quad-0.37889100$

$\begin{array}{lll}-3.01069000 & -1.26669700 & 0.82807900\end{array}$

$-3.38139800-0.81500000-0.61638500$

$\begin{array}{lll}-0.27988500 & 1.94210000 & 1.38134500\end{array}$

$2.76658800 \quad 1.87077800 \quad 0.86496500$

$0.09714000 \quad 1.72762600-1.66056800$

$1.03611700 \quad 2.77113700-0.60668600$

$\begin{array}{lll}4.33831100 & 0.01613800 & 1.35245400\end{array}$

$3.94392700 \quad-2.24258400 \quad 0.38042200$

$1.96295600-2.62608600-1.07871400$

$0.38534300-0.76864100-1.54953800$

$-2.48533700 \quad-2.23316600 \quad 0.81129300$

$-3.90552000 \quad-1.38618900 \quad 1.45067500$

$-3.32484400-1.65153300-1.32328000$

$-4.40884800-0.42220400-0.64844400$

$\begin{array}{lll}-1.24063700 & 2.80693000 & 0.19593200\end{array}$

$\begin{array}{lll}-2.39329300 & 0.24098000 & -0.91768100\end{array}$

$\begin{array}{lll}-2.12983700 & -0.18750000 & 1.31572000\end{array}$

$\begin{array}{lll}-2.42995800 & 0.70528500 & -1.81126300\end{array}$

$\begin{array}{lll}-1.74598900 & -0.24470600 & 2.24549300\end{array}$

\section{D}

$\mathrm{E}=-2469.08321208 \mathrm{au} \quad \mathrm{ZPE}=228.1 \mathrm{kcal} / \mathrm{mol}$

$\begin{array}{lrrr}\mathrm{B} & -1.70970200 & 0.66241000 & 0.26010600 \\ \mathrm{C} & -0.68909100 & 1.86802300 & 0.36372100 \\ \mathrm{C} & 2.58668300 & 0.88516000 & 0.44350500 \\ \mathrm{C} & 0.49110900 & 1.81840700 & -0.64280300 \\ \mathrm{C} & 3.47456300 & -0.16070900 & 0.71831000 \\ \mathrm{C} & 3.25402600 & -1.43059700 & 0.17200500 \\ \mathrm{C} & 2.14001500 & -1.64477500 & -0.64841000 \\ \mathrm{C} & 1.25403000 & -0.59663000 & -0.92094600 \\ \mathrm{C} & 1.46426100 & 0.68426900 & -0.37889100 \\ \mathrm{C} & -3.01069000 & -1.26669700 & 0.82807900 \\ \mathrm{C} & -3.38139800 & -0.81500000 & -0.61638500 \\ \mathrm{H} & -0.27988500 & 1.94210000 & 1.38134500 \\ \mathrm{H} & 2.76658800 & 1.87077800 & 0.86496500 \\ \mathrm{H} & 0.09714000 & 1.72762600 & -1.66056800 \\ \mathrm{H} & 1.03611700 & 2.77113700 & -0.60668600 \\ \mathrm{H} & 4.33831100 & 0.01613800 & 1.35245400 \\ \mathrm{H} & 3.94392700 & -2.24258400 & 0.38042200 \\ \mathrm{H} & 1.96295600 & -2.62608600 & -1.07871400 \\ \mathrm{H} & 0.38534300 & -0.76864100 & -1.54953800 \\ \mathrm{H} & -2.48533700 & -2.23316600 & 0.81129300 \\ \mathrm{H} & -3.90552000 & -1.38618900 & 1.45067500 \\ \mathrm{H} & -3.32484400 & -1.65153300 & -1.32328000 \\ \mathrm{H} & -4.40884800 & -0.42220400 & -0.64844400 \\ \mathrm{H} & -1.24063700 & 2.80693000 & 0.19593200 \\ \mathrm{~N} & -2.39329300 & 0.24098000 & -0.91768100 \\ \mathrm{~N} & -2.12983700 & -0.18750000 & 1.31572000\end{array}$

